\title{
$u-w$ formulation for dynamic problems in large deformation regime solved through an implicit meshfree scheme
}

\author{
Pedro Navas · Lorenzo Sanavia • Susana López-Querol • Rena C. Yu
}

Received: date / Accepted: 25 November 2017

\begin{abstract}
Solving dynamic problems for fluid saturated porous media at large deformation regime is an interesting but complex issue. An implicit time integration scheme is herein developed within the framework of the $u-w$ (solid displacement - relative fluid displacement) formulation for the Biot's equations. In particular, liquid water saturated porous media is considered and the linearization of the linear momentum equations taking into account all the inertia terms for both solid and fluid phases is for the first time presented. The spatial discretization is carried out through a meshfree method, in which the shape functions are based on the principle of local maximum entropy LME. The current methodology is firstly validated with the dynamic consolidation of a soil column and the plastic shear band formulation of a square domain loaded by a rigid footing. The feasibility of this new numerical approach for solving large deformation dynamic problems is finally demonstrated through the application to an embankment problem subjected to an earthquake.
\end{abstract}

Keywords Biot's equations · Meshfree · Implicit schemes $\cdot u-w$ formulation $\cdot$ Hyperelastoplasticity

Pedro Navas and Rena C. Yu

School of Civil Engineering, University of Castilla LaMancha, Avda. Camilo Jose Cela s/n, 13071, Ciudad Real, Spain, E-mail: pedro.navas@uclm.es, rena@uclm.es

Lorenzo Sanavia

Dipartimento di Ingegneria Civile, Edile e Ambientale, Universitá degli Studi di Padova, via F. Marzolo 9, 35131 Padova, Italy, E-mail: lorenzo.sanavia@unipd.it

Susana López-Querol

Department of Civil, Environmental and Geomatic Engineering, University College London, Gower Street, London WC1E 6BT, UK, E-mail: s.lopez-querol@ucl.ac.uk

\section{Introduction}

Predicting the dynamic behavior of saturated porous media at large deformation regime is undoubtedly interesting and complex at the same time. This is evidenced by the very limited literature available in this field $[10,22,27,45]$. It is mainly attributed to the fact that the widely-used displacement-water pressure $u-p_{w}$ formulation for the Biot's equations, though effective for dynamic but low frequency loading [2, 12, 39, 44, 45], is not capable to capture high frequency oscillations for all the permeability [44]. By contrast, the $u-w$ formulation (where $u$ denotes the solid phase displacement and $w$ represents the relative fluid displacement with respect to the solid phase) is particularly suited for solving dynamic and wave propagation problems when the effect of inertia cannot be ignored, since both solid and fluid accelerations are included in the governing equations. In addition, it is more stable than the complete formulation based on the total displacement of the fluid phase, $U$, as nodal unknowns, since the later employs one unique material point for both solid and fluid phases, making it unstable when large deformations occur in the fluid phase $[17,32]$.

Even though explicit solutions for the coupled problem can be found in literature using the total displacement of the water [41], the traditional manner to solve this kind of problems for complete and $u-w$ formulation is through implicit schemes.

Since the derivatives of the governing equations are required, linearization of the terms which are not negligible for large deformation dynamic problems becomes indispensable. However, such complex mathematical operations have not been presented in the literature so far. The most recent advances on this field are made by Sanavia et al. [33] who employed some neglected 
terms of the previous works $[6,7]$ and extended the application of the methodology to unsaturated soils [35] and thermo-mechanical analyses [8]. The current work is the first application of the implicit $u-w$ formulation at large elastic or elasto-plastic strains and it is the first time that the complete Biot's equations are linearized for an implicit Lagrangian scheme.

The mentioned implicit numerical scheme is implemented within a meshfree framework, in particular, the shape functions based on the principle of maximum entropy $[3,31]$ are chosen for spacial discretizations. In addition, the coupling between the solid and fluid phases is carried out through a single set of material points. This is computationally more effective compared to alternative implementations such as those of Material Point Schemes (MPM) [4,9], where two sets of material points were employed. Following the Optimal Transportation Meshfree (OTM) scheme by Li et al. [20], the nodal and material points are reconnected on the fly, which facilitates the simulation of large deformation problems. Regarding the constitutive material models, the modification of the Neo-Hookean material by Ehlers and Eipper [13] for elastic behavior and the DruckerPrager yield criterion for plastic deformation [26] based on [35] are adopted.

The rest of the paper is organized as follows. The $u-w$ equations developed in the deformed configuration are presented next. The employed implicit methodology is developed in Section 3. The constitutive models for the solid behavior are given in Section 4. Applications to various benchmark problems are illustrated in Section 5 whereas the performance of the method in an embankment loaded by an earthquake load is presented in Section 6. Relevant conclusions are drawn in Section 7 .

\section{The $u-w$ formulation for the coupled problem}

For the sake of completeness, we briefly explain here the notations involved for the Biot's equations [5] to solve the coupled problem of a porous medium (solid-fluid mixture). Then the equations for the balance of linear momentum and mass conservation by Lewis and Schrefler [19] are introduced in the spatial setting, which are based on averaging procedures and derived within the Hybrid Mixture Theory. For the kinematic equations the interesting reader can see [19] or [35].

Hereinafter, the vectors and matrices are represented with bold symbols, whereas scalar variables are denoted with regular letters. The symbol ' ' denotes the scalar product between two vectors $\left(\boldsymbol{a} \cdot \boldsymbol{b}=a_{i} b_{i}\right)$, while the symbol ' $:$ ' denotes a double contraction of (adjacent) indices of two tensors of rank two or/and higher (e.g. $\left.\boldsymbol{c}: \boldsymbol{d}=c_{i j} d_{i j}, \boldsymbol{e}: \boldsymbol{f}=e_{i j k l} f_{k l}\right)$. Cartesian coordinates are used throughout.

In the following, $\boldsymbol{u}, \boldsymbol{U}$ and $\boldsymbol{w}$ respectively stand for displacement vectors of the solid skeleton, the absolute displacement of the fluid phase and relative displacement of the fluid phase with respect to the solid one. In addition, $\boldsymbol{w}$ is related with $\boldsymbol{u}$ and $\boldsymbol{U}$ through the porosity, $n$, and the degree of water saturation, $S_{w}$ as follows [23],

$\boldsymbol{w}=n S_{w}(\boldsymbol{U}-\boldsymbol{u})$

It needs to be clarified that $(\boldsymbol{U}-\boldsymbol{u})$ is often coined as $\boldsymbol{u}^{w s}$ in the literature [19]. In Eq. (1), the porosity is calculated as

$n=\frac{d v_{h}}{d v_{h}+d v_{s}}$,

where $d v_{h}$ and $d v_{s}$ are the volumes of the voids and solid grains in the deformed configuration respectively. Note that in the current work, totally saturated porous medium is assumed, i.e., $d v_{h}$ coincides with the water volume, which results in $S_{w}$ equal to 1 .

The parameters $n$ and $S_{w}$ also map out the mixture density, $\rho$, with that of the fluid and solid particles, $\rho_{w}$ and $\rho_{s}$, as follows

$\rho=(1-n) \rho_{s}+n S_{w} \rho_{w}$.

Likewise, the volumetric compressibility of the mixture, $Q$, is related with the bulk modulus of the solid grains, $K_{s}$, and the compressive modulus of the fluid phase (water), $K_{w}$, [43] i.e.,

$Q=\left[\frac{\alpha-n}{K_{s}}+\frac{n}{K_{w}}\right]^{-1}$.

where $\alpha$ is the Biot's coefficient:

$\alpha=1-\frac{K}{K_{s}}$

being $K$ the bulk modulus of the solid skeleton. For soils, since the solid grains are much less compressible than the porous skeleton, $\alpha$ can be considered equal to 1 .

With respect to the sign criterion for stresses and strains, tensile ones are assumed positive, except for the pore pressure, $p_{w}$, which is negative for tension.

The Terzaghi's effective stress [38], $\boldsymbol{\sigma}^{\prime}$, is related with the total Cauchy stress tensor, $\boldsymbol{\sigma}$, and the pore pressure, $p_{w}$, as follows,

$\boldsymbol{\sigma}^{\prime}=\boldsymbol{\sigma}+\alpha p_{w} \mathbf{I}$

where $\mathbf{I}$ is the second order unit tensor. 
2.1 Systems of equations for the linear momentum balance

On the one hand, starting from the general form of Darcy's law for any fluid phase, the system equations of the linear momentum balance for saturated soil according to Lewis and Schrefler [19] are expressed as follows,

$n S_{w} \boldsymbol{v}^{w s}=\frac{\boldsymbol{k}}{\mu_{w}}\left[-\operatorname{grad} p_{w}+\rho_{w}\left(\boldsymbol{g}-\boldsymbol{a}^{s}-\boldsymbol{a}^{w s}\right)\right]$

where, as previously mentioned, the degree of saturation, $S_{w}$, is equal to one; the relative velocity of the fluid, $\boldsymbol{v}^{w s}$, is defined as $\dot{\boldsymbol{w}} / n$, where $\dot{\square}$ represents the material time derivative of $\square$ with respect to the solid; $\boldsymbol{a}^{s} \equiv \ddot{\boldsymbol{u}}$ denotes the solid phase acceleration, $\boldsymbol{a}^{w s}=$ $\ddot{\boldsymbol{w}} / n$ is the relative acceleration of the fluid respect to the solid phase, $\boldsymbol{g}$ represents the external acceleration vector, $\mu_{w}$ denotes the dynamic viscosity of the water and $\boldsymbol{k}$ is the intrinsic permeability tensor, which becomes a unit tensor multiplied by the scalar $k$, intrinsic permeability, when isotropic permeability is assumed. Consequently, Eq. (7) can be re-written as

$-\operatorname{grad} p_{w}-\frac{\mu_{w}}{k} \dot{\boldsymbol{w}}+\rho_{w}\left(\boldsymbol{g}-\ddot{\boldsymbol{u}}-\frac{\ddot{\boldsymbol{w}}}{n}\right)=\mathbf{0}$

The relationship $\boldsymbol{v}^{w s}=\dot{\boldsymbol{w}} / n$ has been derived from Eq. (1) computing the material time derivative of $\boldsymbol{w}$ with respect to the solid and neglecting the material time derivative of the porosity with respect to the solid for simplicity.

On the other hand, Lewis and Schrefler [19] also give the linear momentum balance equation for the multiphase system under saturated conditions as the summation of the dynamic equations for the individual constituents relative to the solid as

$\operatorname{div} \boldsymbol{\sigma}-\rho \boldsymbol{a}^{s}-n \rho_{w} \boldsymbol{a}^{w s}+\rho \boldsymbol{g}=\mathbf{0}$.

Taking into account Eq. (6), we obtain the system equations for the linear momentum balance as

$\operatorname{div}\left[\boldsymbol{\sigma}^{\prime}-\alpha p_{w} \mathbf{I}\right]-\rho \ddot{\boldsymbol{u}}-\rho_{w} \ddot{\boldsymbol{w}}+\rho \boldsymbol{g}=\mathbf{0}$.

Both linear momentum balance equations of the mixture and the fluid were presented by Zienkiewicz et al. [43] with the convective terms, which can be neglected in the present research as the vorticity is relatively small compared to the rest of the terms.

\subsection{Mass balance equation}

The mass balance equation of the liquid water in a isothermal totally saturated media with compressible grains and water is [19]

$\left(\frac{\alpha-n}{K_{s}}+\frac{n}{K_{w}}\right) \dot{p_{w}}+\alpha \operatorname{div}(\dot{\boldsymbol{u}})+\frac{\operatorname{div}\left(\rho_{w} \dot{\boldsymbol{w}}\right)}{\rho_{w}}=0$

Taking into consideration Eq. (4) and considering constant water density and $\alpha$ equal to 1 we obtain the mass balance equation as follows:

$\frac{\dot{p}_{w}}{Q}+\operatorname{div} \dot{\boldsymbol{u}}+\operatorname{div} \dot{\boldsymbol{w}}=0$.

The above equation can be integrated over time to obtain the pore pressure as

$p_{w}=-Q[\operatorname{div}(\boldsymbol{u})+\operatorname{div}(\boldsymbol{w})]+p_{w_{0}}$,

where $p_{w_{0}}$ is the initial pore pressure.

It needs to be pointed out that, if accelerations of the solid and the fluid are negligible, as in a quasi static $u-p_{w}$ formulation, and the solid grain and the fluid can be considered incompressible, substituting Darcy's law into Eq. (12), the liquid water mass balance equation can be simplified as $[34,35]$

$\operatorname{div} \dot{\boldsymbol{u}}+\operatorname{div}\left[\frac{\boldsymbol{k}}{\mu_{w}}\left(-\operatorname{grad} p_{w}+\rho_{w} \boldsymbol{g}\right)\right]=\mathbf{0}$.

2.3 The weak form of system equations for the $u-w$ formulation

The weak form of the system equations for the $u-w$ formulation is obtained applying the principle of virtual displacements to the linear momentum equation of both the solid and fluid phases, Eqs. (8) and (10).

Let $\delta \boldsymbol{u}$ represent the virtual displacement vector for the solid phase, the weak form of the linear momentum balance equation (10) reads

$\int_{B}\left[\operatorname{div}(\boldsymbol{\sigma})-\rho \ddot{\boldsymbol{u}}-\rho_{w} \ddot{\boldsymbol{w}}+\rho \boldsymbol{g}\right] \cdot \delta \boldsymbol{u} d v=0$

where $B$ is the volume of the spatial domain. Applying the Green's Theorem to Eq. (15), we obtain

$$
\begin{aligned}
& -\int_{B} \boldsymbol{\sigma}: \operatorname{grad}(\delta \boldsymbol{u}) d v+\int_{B}\left[-\rho \ddot{\boldsymbol{u}}-\rho_{w} \ddot{\boldsymbol{w}}+\rho \boldsymbol{g}\right] \cdot \delta \boldsymbol{u} d v \\
& +\int_{\delta B} \overline{\boldsymbol{t}} \cdot \delta \boldsymbol{u} d s=0 .
\end{aligned}
$$

being $\delta B$ the boundary where the traction $\overline{\boldsymbol{t}}$ is applied. Taking into account Terzaghi's definition of the effective stress and mass conservation, the terms in Eq. (16) can 
be arranged to yield the final expression of the weak form for the mixture as follows

$$
\begin{aligned}
& -\int_{B} \boldsymbol{\sigma}^{\prime}: \operatorname{grad}(\delta \boldsymbol{u}) d v-\int_{B} Q \operatorname{div}(\boldsymbol{u}) \boldsymbol{I}: \operatorname{grad}(\delta \boldsymbol{u}) d v \\
& -\int_{B} Q \operatorname{div}(\boldsymbol{w}) \boldsymbol{I}: \operatorname{grad}(\delta \boldsymbol{u}) d v \\
& +\int_{B}\left[-\rho \ddot{\boldsymbol{u}}-\rho_{w} \ddot{\boldsymbol{w}}+\rho \boldsymbol{g}\right] \cdot \delta \boldsymbol{u} d v+\int_{\delta B} \overline{\boldsymbol{t}} \cdot \delta \boldsymbol{u} d s=0
\end{aligned}
$$

Let $\delta \boldsymbol{w}$ stand for the virtual displacements for the fluid phase, the corresponding weak form of the linear momentum equation (8) is written as

$$
\int_{B}\left[-\operatorname{grad}\left(p_{w}\right)-\frac{\mu_{w}}{k} \dot{\boldsymbol{w}}+\rho_{w}\left(\boldsymbol{g}-\ddot{\boldsymbol{u}}-\frac{\ddot{\boldsymbol{w}}}{n}\right)\right] \cdot \delta \boldsymbol{w} d v=0
$$

Applying the Green's Theorem to the above equation, we have

$$
\begin{aligned}
& \int_{B} p_{w} \boldsymbol{I}: \operatorname{grad}(\delta \boldsymbol{w}) d v-\int_{B} \frac{\mu_{w}}{k} \dot{\boldsymbol{w}} \cdot \delta \boldsymbol{w} d v+ \\
& \int_{B} \rho_{w}\left(\boldsymbol{g}-\ddot{\boldsymbol{u}}-\frac{\ddot{\boldsymbol{w}}}{n}\right) \cdot \delta \boldsymbol{w} d v-\int_{\delta B} \overline{\boldsymbol{t}}_{w} \cdot \delta \boldsymbol{w} d s=0 .
\end{aligned}
$$

being $\overline{\boldsymbol{t}}_{w}$ the traction of the fluid phase. Taking into account Eq. (13), the final weak form of the linear momentum equation of the liquid water is obtained as follows,

$$
\begin{aligned}
& -\int_{B} Q \operatorname{div}(\boldsymbol{u}) \operatorname{div}(\delta \boldsymbol{w}) d v-\int_{B} Q \operatorname{div}(\boldsymbol{w}) \operatorname{div}(\delta \boldsymbol{w}) d v \\
& -\int_{B} \frac{\mu_{w}}{k} \dot{\boldsymbol{w}} \cdot \delta \boldsymbol{w} d v-\int_{B} \ddot{\boldsymbol{w}} \frac{\rho_{w}}{n} \cdot \delta \boldsymbol{w} d v \\
& +\int_{B} \rho_{w}(\boldsymbol{g}-\ddot{\boldsymbol{u}}) \cdot \delta \boldsymbol{w} d v-\int_{\delta B} \overline{\boldsymbol{t}}_{w} \cdot \delta \boldsymbol{w} d s=0
\end{aligned}
$$

\section{Time and spatial discretization and consistent linearization}

In this Section, we describe in detail the implicit time integration scheme, including the linearization process and the Newton-Raphson algorithm and the meshfree spatial discretization based on LME shape functions.

\subsection{Implicit solution and Newton-Raphson algorithm}

As mentioned before, the framework of the $u-w$ formulation, also known as the complete formulation (since no additional assumption is required), each node contains both solid and fluid degrees of freedom, $\boldsymbol{u}$ and $\boldsymbol{w}$, whereas the pore pressure, $p_{w}$, is calculated at the
Table 1 The $\alpha$-parameters of the Newmark scheme.

$$
\begin{array}{ll}
\alpha_{1}=\frac{1}{\beta \Delta t^{2}} & \alpha_{2}=\frac{1}{\beta \Delta t} \\
\alpha_{3}=\frac{1}{2 \beta}-1 & \alpha_{4}=\frac{\gamma}{\beta \Delta t} \\
\alpha_{5}=1-\frac{\gamma}{\beta} & \alpha_{6}=\left(1-\frac{\gamma}{2 \beta}\right) \Delta t \\
\alpha_{7}=1 & \alpha_{8}=1
\end{array}
$$

material point afterwards. By contrast, in the more traditional $u-p_{w}$ formulation, $p_{w}$ is considered directly as an additional nodal unknown. Consequently, the imposition of impervious boundary conditions is a bit more complicated.

In a two-dimensional problem, the nodal unknowns can be written as:

$$
\boldsymbol{u}^{*}=\left[\begin{array}{llll}
u_{x} & u_{y} & w_{x} & w_{y}
\end{array}\right]^{T} .
$$

After assembling the elementary matrices, the final system of equations can be simplified as

$\boldsymbol{R}_{k+1}+\boldsymbol{C} \dot{\boldsymbol{u}}_{k+1}+\boldsymbol{M} \ddot{\boldsymbol{u}}_{k+1}=\boldsymbol{P}_{k+1}$,

where $\boldsymbol{R}, \boldsymbol{C}$ and $\boldsymbol{M}$ respectively denote the internal forces vector and damping and mass matrices, whereas $\boldsymbol{P}$ is the external forces vector, which contains both gravity acceleration and external nodal forces. $k+1$ represents the current step.

In order to solve Eq. (21) in an implicit way, the Newmark equations are written in terms of the incremental displacements, i.e.

$\ddot{\boldsymbol{u}}_{k+1}=\alpha_{1} \Delta \boldsymbol{u}_{k+1}-\alpha_{2} \dot{\boldsymbol{u}}_{k}-\alpha_{3} \ddot{\boldsymbol{u}}_{k}$,

$\dot{\boldsymbol{u}}_{k+1}=\alpha_{4} \Delta \boldsymbol{u}_{k+1}+\alpha_{5} \dot{\boldsymbol{u}}_{k}+\alpha_{6} \ddot{\boldsymbol{u}}_{k}$,

where the $\alpha$-parameters are listed in Table 1 according to Wriggers [40]. These coefficients can be easily extended to any other time integration schemes.

In the current work, solutions are obtained with a traditional Newmark time integration scheme with $\gamma=0.6$ and $\beta=0.325$.

Inserting Eqs. (22-23) to Eq. (21), the equations for the unknowns can be re-written as:

$$
\begin{aligned}
\boldsymbol{G}_{k+1} & =\boldsymbol{M}\left[\alpha_{1} \Delta \boldsymbol{u}_{k+1}-\alpha_{2} \dot{\boldsymbol{u}}_{k}-\alpha_{3} \ddot{\boldsymbol{u}}_{k}\right] \\
& +\boldsymbol{C}\left[\alpha_{4} \Delta \boldsymbol{u}_{k+1}+\alpha_{5} \dot{\boldsymbol{u}}_{k}+\alpha_{6} \ddot{\boldsymbol{u}}_{k}\right] \\
& +\alpha_{7} \boldsymbol{R}_{k+1}-\boldsymbol{P}_{k}-\alpha_{8} \Delta \boldsymbol{P}_{k+1}=\mathbf{0},
\end{aligned}
$$

or in the compact form:

$$
\boldsymbol{G}(\chi, \boldsymbol{\eta})=\mathbf{0},
$$

where $\quad \chi=\left[\chi^{\boldsymbol{u}}, \boldsymbol{\chi}^{\boldsymbol{w}}\right]^{T} \quad$ is the deformation mapping

$$
\text { and } \boldsymbol{\eta}=[\delta \boldsymbol{u}, \delta \boldsymbol{w}]^{T}, \Delta \boldsymbol{u}^{*}=[\Delta \boldsymbol{u}, \Delta \boldsymbol{w}]^{T} .
$$

To solve the above non-linear equations, Taylor's series are employed in the current configuration. After the linearization of $\chi$, Eq. (25) becomes

$$
\boldsymbol{G}\left(\overline{\boldsymbol{\chi}}, \boldsymbol{\eta}, \Delta \boldsymbol{u}^{*}\right)_{k+1}^{i+1} \cong
$$

$\boldsymbol{G}(\overline{\boldsymbol{\chi}}, \boldsymbol{\eta})_{k+1}^{i}+D \boldsymbol{G}(\overline{\boldsymbol{\chi}}, \boldsymbol{\eta})_{k+1}^{i} \cdot \Delta \boldsymbol{u}_{k+1}^{* i+1} \cong \mathbf{0}$, 
where $\bar{\chi}$ is the linearized deformation mapping. Taking into account the fact that operator $\boldsymbol{G}$ is composed in two parts, the derivatives taking the following form:

$D \boldsymbol{G} \cdot \Delta \boldsymbol{u}^{*}=\left[\begin{array}{c}D G_{L M S} \cdot \Delta \boldsymbol{u}+D G_{L M S} \cdot \Delta \boldsymbol{w} \\ D G_{L M W} \cdot \Delta \boldsymbol{u}+D G_{L M W} \cdot \Delta \boldsymbol{w}\end{array}\right]$,

where $D G_{L M S}$ and $D G_{L M W}$ represent the derivative of the linear momentum equations Eq. (17) and Eq. (20), of the solid phase and the fluid phase respectively.

According to Wriggers [40], any Newton method can be applied to determine the unknown displacements. We first calculate the tangential stiffness matrix, i.e.

$\boldsymbol{K}\left(\boldsymbol{u}_{k+1}^{i}\right)=\boldsymbol{K}_{k+1}^{i}=\left.\frac{\partial \boldsymbol{R}}{\partial \boldsymbol{u}}\right|_{\boldsymbol{u}_{k+1}^{i}}$.

that allows us to solve the system equations in an iterative manner (iteration index $i$ ). The iteration finishes when $\boldsymbol{G}_{k+1}^{i}$ is lower than a given tolerance:

$$
\begin{aligned}
{\left[\alpha_{1} \boldsymbol{M}+\alpha_{4} \boldsymbol{C}+\alpha_{7} \boldsymbol{K}_{k+1}^{i}\right] \Delta \boldsymbol{u}_{k+1}^{i+1} } & =-\boldsymbol{G}\left(\boldsymbol{u}_{k+1}^{i}\right), \\
\text { where } \boldsymbol{u}_{k+1}^{i+1} & =\boldsymbol{u}_{k+1}^{i}+\Delta \boldsymbol{u}_{k+1}^{i+1} .
\end{aligned}
$$

Rewritting Eq. (24) to separate the current $(k+1)$ and previous $(k)$ terms, since the later ones are not susceptible of linearization as they are constants that come from the previous step, we have

$$
\begin{aligned}
\boldsymbol{G}_{k+1} & =\left[\alpha_{1} \boldsymbol{M}+\alpha_{4} \boldsymbol{C}\right] \boldsymbol{u}_{k+1}+\alpha_{7} \boldsymbol{R}\left(\boldsymbol{u}_{k+1}\right)-\alpha_{8} \boldsymbol{P}_{k+1} \\
& +\left[\alpha_{1} \boldsymbol{M} \boldsymbol{u}_{k}-\alpha_{2} \boldsymbol{M} \dot{\boldsymbol{u}}_{k}-\alpha_{3} \boldsymbol{M} \ddot{\boldsymbol{u}}_{k}\right] \\
& +\left[\alpha_{4} \boldsymbol{C} \boldsymbol{u}_{k}+\alpha_{5} \boldsymbol{C} \dot{\boldsymbol{u}}_{k}+\alpha_{6} \boldsymbol{C} \ddot{\boldsymbol{u}}_{k}\right]-\alpha_{f} \boldsymbol{P}_{k} \\
& =\left[\alpha_{1} \boldsymbol{M}+\alpha_{4} \boldsymbol{C}\right] \boldsymbol{u}_{k+1}+\alpha_{7} \boldsymbol{R}\left(\boldsymbol{u}_{k+1}\right) \\
& -\alpha_{8} \boldsymbol{P}_{k+1}+\boldsymbol{F}_{k}^{i n t} .
\end{aligned}
$$

Consequently, after integration in time, Eq. (17) and Eq. (20) are written at time $k+1$ and transformed as follows

$$
\begin{aligned}
& -\alpha_{7} \int_{B} \boldsymbol{\sigma}^{\prime}: \operatorname{grad}(\delta \boldsymbol{u}) d v-\alpha_{7} \int_{B} Q \operatorname{div}(\boldsymbol{u}) \operatorname{div}(\delta \boldsymbol{u}) d v \\
& -\alpha_{7} \int_{B} Q \operatorname{div}(\boldsymbol{w}) \operatorname{div}(\delta \boldsymbol{u}) d v-\alpha_{1} \int_{B}\left[\rho \boldsymbol{u}+\rho_{w} \boldsymbol{w}\right] \cdot \delta \boldsymbol{u} d v \\
& +\alpha_{8} \int_{B} \rho \boldsymbol{g} \cdot \delta \boldsymbol{u} d v+\alpha_{8} \int_{\delta B} \overline{\boldsymbol{t}} \cdot \delta \boldsymbol{u} d s=\mathbf{0} \\
& -\int_{B} \alpha_{7} Q \operatorname{div}(\boldsymbol{u}) \operatorname{div}(\delta \boldsymbol{w}) d v-\int_{B} \alpha_{7} Q \operatorname{div}(\boldsymbol{w}) \operatorname{div}(\delta \boldsymbol{w}) d v \\
& -\alpha_{4} \int_{B} \frac{\mu_{w}}{k} \boldsymbol{w} \cdot \delta \boldsymbol{w} d v-\alpha_{1} \int_{B} \frac{\rho_{w}}{n} \boldsymbol{w} \cdot \delta \boldsymbol{w} d v \\
& -\alpha_{1} \int_{B} \rho_{w} \boldsymbol{u} \cdot \delta \boldsymbol{w} d v+\alpha_{8} \int_{B} \rho_{w} \boldsymbol{g} \cdot \delta \boldsymbol{w} d v \\
& -\alpha_{8} \int_{\delta B} \overline{\boldsymbol{t}}_{w} \cdot \delta \boldsymbol{w} d s=\mathbf{0} .
\end{aligned}
$$

The results of the linearization process for Eq. (31) and Eq.(32) are given in Eq. (33) and Eq. (34) respectively, whereas the details are described in Appendix A.
- Linear momentum of for the solid phase

$$
\begin{aligned}
& -\alpha_{7} \int_{B} \operatorname{grad}(\delta \boldsymbol{u}): \boldsymbol{c}^{e p}: \operatorname{grad}(\Delta \boldsymbol{u}) d v \\
& -\alpha_{7} \int_{B} \boldsymbol{\sigma}^{\prime}: \operatorname{grad}^{T}(\delta \boldsymbol{u}) \operatorname{grad}(\Delta \boldsymbol{u}) d v \\
& -\alpha_{7} \int_{B} \operatorname{grad}(\delta \boldsymbol{u}):(Q[\operatorname{div}(\Delta \boldsymbol{u})+\operatorname{div}(\Delta \boldsymbol{w})] \boldsymbol{I}) d v \\
& -\alpha_{7} \int_{B} \operatorname{grad}(\delta \boldsymbol{u}): p_{w} \operatorname{grad}^{T}(\Delta \boldsymbol{u}) d v \\
& -\alpha_{7} \int_{B} \operatorname{grad}(\delta \boldsymbol{u}): p_{w} \frac{1-n}{n} \operatorname{div}(\Delta \boldsymbol{u}) \boldsymbol{I} d v \\
& -\alpha_{1} \int_{B} \delta \boldsymbol{u} \cdot\left[\rho \Delta \boldsymbol{u}+\rho_{w} \Delta \boldsymbol{w}+\rho_{w} \operatorname{div}(\Delta \boldsymbol{u})(\boldsymbol{u}+\boldsymbol{w})\right] d v \\
& +\alpha_{8} \int_{B} \rho_{w} \delta \boldsymbol{u} \cdot \boldsymbol{g} \operatorname{div}(\Delta \boldsymbol{u}) d v
\end{aligned}
$$

- Linear momentum for the fluid phase:

$$
\begin{aligned}
& -\alpha_{7} \int_{B} \operatorname{grad}(\delta \boldsymbol{w}):(Q[\operatorname{div}(\Delta \boldsymbol{u})+\operatorname{div}(\Delta \boldsymbol{w})] \boldsymbol{I}) d v \\
& -\alpha_{7} \int_{B} \operatorname{grad}(\delta \boldsymbol{w}): p_{w} \operatorname{grad}^{T}(\Delta \boldsymbol{u}) d v \\
& -\alpha_{7} \int_{B} \operatorname{grad}(\delta \boldsymbol{w}): p_{w} \frac{1-n}{n} \operatorname{div}(\Delta \boldsymbol{u}) \boldsymbol{I} d v \\
& -\alpha_{4} \int_{B} \frac{\mu_{w}}{k} \delta \boldsymbol{w} \cdot\left[\Delta \boldsymbol{w}+\operatorname{div}(\Delta \boldsymbol{u})\left(1-\frac{1-n}{k} \frac{\partial k}{\partial n}\right) \boldsymbol{w}\right] d v \\
& -\alpha_{1} \int_{B} \frac{\rho_{w}}{n} \delta \boldsymbol{w} \cdot\left[\Delta \boldsymbol{w}+\frac{2 n-1}{n} \operatorname{div}(\Delta \boldsymbol{u}) \boldsymbol{w}\right] d v \\
& -\alpha_{1} \int_{B} \rho_{w} \delta \boldsymbol{w} \cdot[\Delta \boldsymbol{u}+\operatorname{div}(\Delta \boldsymbol{u}) \boldsymbol{u}] d v \\
& +\alpha_{8} \int_{B} \rho_{w} \delta \boldsymbol{w} \cdot \boldsymbol{g} \operatorname{div}(\Delta \boldsymbol{u}) d v
\end{aligned}
$$

\subsection{Spatial discretization}

The shape function employed is based that of Arroyo and Ortiz [3], who defined exponential functions based on the principle of the local maximum entropy (LME). For a node $a$, it reads,

$N_{a}(\mathbf{x})=\frac{\exp \left[-\beta\left|\mathbf{x}-\mathbf{x}_{\mathbf{a}}\right|^{2}+\boldsymbol{\lambda}^{*} \cdot\left(\mathbf{x}-\mathbf{x}_{\mathbf{a}}\right)\right]}{Z\left(\mathbf{x}, \boldsymbol{\lambda}^{*}(\mathbf{x})\right)}$,

where

$Z(\mathbf{x}, \boldsymbol{\lambda})=\sum_{a=1}^{N_{b}} \exp \left[-\beta\left|\mathbf{x}-\mathbf{x}_{\mathbf{a}}\right|^{2}+\boldsymbol{\lambda} \cdot\left(\mathbf{x}-\mathbf{x}_{\mathbf{a}}\right)\right]$

$N_{b}$ representes the neighborhood size. The parameter $\beta$ defines the shape of the neighborhood and $\boldsymbol{\lambda}^{*}(\mathbf{x})$ comes from the minimization of the function $g(\boldsymbol{\lambda})=$ 
$\log Z(\mathbf{x}, \boldsymbol{\lambda})$ to guarantee the maximum entropy. The first derivatives of the shape function are then obtained from differentiating the shape function itself to get the Hessian matrix $\mathbf{J}$ in the following expression:

$\nabla N_{a}^{*}=-N_{a}^{*}\left(\mathbf{J}^{*}\right)^{-1}\left(\mathbf{x}-\mathbf{x}_{\mathbf{a}}\right)$.

A modified Nelder-Mead algorithm developed by Navas et al. [26] is used for the minimization process in the current work.

\section{Material models for the solid phase}

Since the first term of Eq. (33) contains $\boldsymbol{c}^{e p}$, which depends on specific material models, we illustrate in this Section the constitutive models employed for the solid phase based on hyperelasticity or hyperelastoplasticity.

\subsection{Elastic material model: the Neo-Hookean solid}

In order to predict the non-linear elastic behavior for solids undergoing large deformations and to be able to take into consideration the effect of the compaction point, the variation of the volume and the initial porosity in soils, the modified Neo-hookean material model proposed by Ehlers and Eipper [13] is adopted. It reads as follows,

$\boldsymbol{\tau}_{k+1}^{\prime}=G\left(\boldsymbol{b}_{k+1}-\mathbf{I}\right)+\lambda n_{0}^{2}\left(\frac{J}{n_{0}}-\frac{J}{J-1+n_{0}}\right) \mathbf{I}$,

where $\boldsymbol{\tau}^{\prime}$ is the effective Kirchhoff stress tensor $\left(\boldsymbol{\tau}^{\prime}=\right.$ $\left.J \boldsymbol{\sigma}^{\prime}\right), J$ represents the Jacobian determinant of the deformation gradient at step $k+1, \boldsymbol{b}$ is the left CauchyGreen tensor, $n_{0}$ is the initial porosity, $G$ and $\lambda$ are the Lamé constants. Consequently, the continuous variation of the tangent moduli, $\boldsymbol{c}^{e}$, is depicted as

$$
\begin{aligned}
\boldsymbol{c}^{e}= & 2\left[G-\lambda n_{0} J \frac{J-1}{J+n_{0}-1}\right] \mathbf{1} \\
\lambda & {\left[n_{0} J \frac{J^{2}+\left(1-n_{0}\right)(1-2 J)}{\left(J+n_{0}-1\right)^{2}}\right](\mathrm{I} \otimes \mathrm{I}), }
\end{aligned}
$$

where $\mathbf{1}$ and I represent the respective fourth and second order unit tensors.

4.2 Elasto-plastic material model: the Drucker-Prager yield criterion

The elasto-plastic behavior of the solid skeleton at finite strains is based on the multiplicative decomposition of the deformation gradient of the solid $\boldsymbol{F}^{s}$ into an elastic and plastic part originally proposed by Lee [18] for crystals

$\boldsymbol{F}^{s}=\boldsymbol{F}^{s e} \boldsymbol{F}^{s p}$
Table 2 Parameters for the Drucker-Prager (DP) yield criteria

\begin{tabular}{lll}
\hline & DP: Plane strain & DP: Outer cone \\
\hline$\alpha_{F}$ & $\frac{\tan \phi}{\sqrt{\left(3+4 \tan ^{2} \phi\right)}} \sqrt{\frac{2}{3}}$ & $\frac{2 \sin \phi}{3-\sin \phi} \sqrt{\frac{2}{3}}$ \\
$\alpha_{Q}$ & $\frac{\tan \psi}{\sqrt{\left(3+4 \tan ^{2} \psi\right)}} \sqrt{\frac{2}{3}}$ & $\frac{2 \sin \psi}{3-\sin \psi} \sqrt{\frac{2}{3}}$ \\
$\beta$ & $\frac{3}{\sqrt{\left(3+4 \tan ^{2} \phi\right)}} \sqrt{\frac{2}{3}}$ & $\frac{6 \cos \phi}{3-\sin \phi} \sqrt{\frac{2}{3}}$ \\
\hline
\end{tabular}

This decomposition states the existence of an intermediate stress free configuration and its validity has been suggested for cohesive-frictional soils by NematNasser [30]. The elasto-plastic behavior is assumed isotropic. The constitutive equations, their algorithmic formulation based on the product formula algorithm proposed for the single phase material by Simo [37] and the return mapping and the consistent tangent operator can be found in [35]. Here it is recalled that the formulation is written in terms of the effective Kirchhoff stress tensor and the logarithmic principal values of the elastic left Cauchy-Green strain tensor. The yield function restricting the stress state is developed in the form of DruckerPrager, to take into account the dilatant/contractant behavior of dense or loose sands, respectively. The singular behavior of the Drucker-Prager yield surface in the zone of the apex is solved using the concept of multisurface plasticity.

This approach is selected due to its computational efficiency, because, by defining a limit pressure, $p^{\text {lim }}$, given by Eq. (41), the location of a given stress state (on the cone or over the apex) can be determined before the plastic strain is known.

$$
\begin{aligned}
p_{\text {lim }} & =\frac{3 \alpha_{Q} K}{2 G}\left\|\mathbf{s}_{k+1}^{\text {trial }}\right\| \\
& +\frac{\beta}{3 \alpha_{F}}\left(\frac{\left\|\mathbf{s}_{k+1}^{\text {trial }}\right\|}{2 G} H \sqrt{1+3 \alpha_{Q}^{2}}+c_{k}\right),
\end{aligned}
$$

where $K$ and $G$ are the bulk and shear modulus, $c_{k+1}$ is the current cohesion and its derivative, $H$, is the hardening modulus; $\beta$ and $\alpha_{F}$ are Drucker-Prager parameters related to the friction angle, $\phi$, whereas $\alpha_{Q}$ depends on the dilatancy angle, $\psi$. These parameters are illustrated in Tab. 2. In addition, $\left\|\mathbf{s}_{k+1}^{\text {trial }}\right\|$ represents the norm of the deviatoric trial stress tensor in the time step $k+1, \mathbf{s}_{k+1}^{\text {trial }}$, calculated from pressure and the trial logarithmic strain $[11,35]$. Once the stress state is located, the yield condition of either classical $\left(\Phi^{c l}\right)$ or apex $\left(\Phi^{a p}\right)$ region is used.

$$
\begin{array}{r}
\Phi^{c l}=\left\|\mathbf{s}_{k+1}^{\text {trial }}\right\|-2 G \Delta \gamma+3 \alpha_{F}\left[p_{k+1}^{\text {trial }}-\right. \\
\left.-3 K \alpha_{Q} \Delta \gamma\right]-\beta c_{k+1},
\end{array}
$$




$$
\begin{aligned}
\Phi^{a p}= & \frac{\beta}{3 \alpha_{F}}\left[c_{k}+H \sqrt{\Delta \gamma_{1}^{2}+3 \alpha_{Q}^{2}\left(\Delta \gamma_{1}+\Delta \gamma_{2}\right)^{2}}\right] \\
& -p_{k+1}^{\text {trial }}+3 K \alpha_{Q}\left(\Delta \gamma_{1}+\Delta \gamma_{2}\right) .
\end{aligned}
$$

where $\Delta \gamma_{1}=\frac{\left\|\mathbf{s}_{k+1}^{\text {tral }}\right\|}{2 G}, \Delta \gamma$ and $\Delta \gamma_{2}$ are the objective functions to be calculated in the Newton-Raphson scheme for the classical or apex regions accordingly.

The above implementation, recently tested for explicit integrations within the framework of Optimal Transportation Meshfree schemes [26], is herein extended for implicit time integrations. According to Sanavia et al [35], depending on where the current stress state is located, the corresponding tangent moduli are calculated as:

- Non-corner zone:

$$
\begin{aligned}
\boldsymbol{c}^{e p}= & K\left[1-\frac{9 \alpha_{Q} \alpha_{F} K}{c_{1}}\right](\mathrm{I} \otimes \mathrm{I}) \\
& +2 G\left[1-\frac{2 G \Delta \gamma}{\left\|\mathbf{s}_{k+1}^{t i a l}\right\|}\right]\left(\mathbf{1}-\frac{1}{3} \mathrm{I} \otimes \mathrm{I}\right) \\
& -\frac{6 \alpha_{Q} K G}{c_{1}}\left(\boldsymbol{I} \otimes \boldsymbol{n}_{k+1}^{t r}\right)-\frac{6 \alpha_{F} K G}{c_{1}}\left(\boldsymbol{n}_{k+1}^{t r} \otimes \mathrm{I}\right) \\
& -4 G^{2}\left[\frac{1}{c_{1}}-\frac{\Delta \gamma}{\left\|\mathbf{s}_{k+1}^{t r i a l}\right\|}\right]\left(\boldsymbol{n}_{k+1}^{t r} \otimes \boldsymbol{n}_{k+1}^{t r}\right),
\end{aligned}
$$

where

$$
c_{1}=9 \alpha_{F} \alpha_{Q} K+2 G+\beta H \sqrt{\left.1+3 \alpha_{Q}^{2}\right]} .
$$

- Corner zone:

$$
\boldsymbol{c}^{e p}=K c_{2}(\mathrm{I} \otimes \mathrm{I})+\frac{K c_{2}}{2 \alpha_{Q} G \Delta \gamma_{T}}\left(\mathrm{I} \otimes \boldsymbol{s}_{k+1}^{t r}\right),
$$

where

$$
c_{2}=\frac{\alpha_{Q} \beta H \Delta \gamma_{T}}{3 \alpha_{Q} K \sqrt{\Delta \gamma_{1}^{2}+3 \alpha_{Q}^{2} \Delta \gamma_{T}^{2}}+\alpha_{Q} \beta H \Delta \gamma_{T}},
$$

and

$$
\Delta \gamma_{T}=\Delta \gamma_{1}+\Delta \gamma_{2} .
$$

In the above equations, $\boldsymbol{n}_{k+1}^{t r}$ is the normalized unit tensor of the trial deviatoric stress tensor, $\boldsymbol{s}_{k+1}^{t r}$, i.e.,

$\boldsymbol{n}_{k+1}^{t r}=\frac{\boldsymbol{s}_{k+1}^{t r}}{\left\|\mathbf{s}_{k+1}^{t r}\right\|}$.

\section{Validation}

In this Section, we validate the developed implicit Lagrangian scheme through three benchmark problems. The first one is the elastic dynamic consolidation of a soil column proposed by Zienkiewicz et al. [44] to assess the performance of the complete formulation when high frequencies are involved. The second one is a large deformation consolidation of the same soil column. The third one deals with the formation of a plastic shear band.

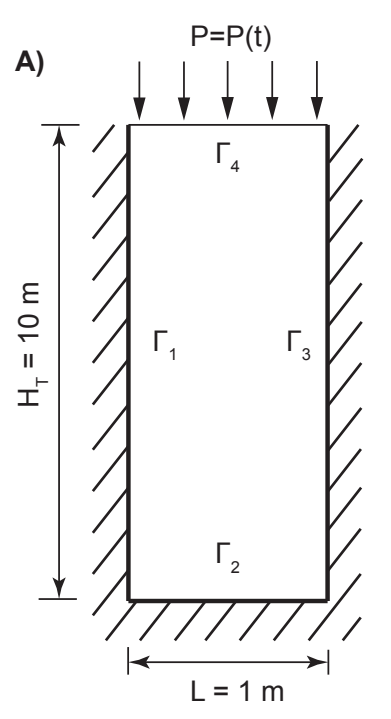

B) Dynamic consolidation

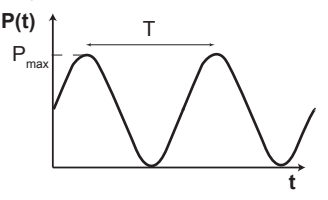

C) Large deformation consolidation

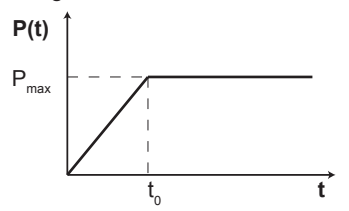

$\Gamma_{1}: u_{x}=0, w_{x}=0$

$\Gamma_{2}: u_{y}=0, w_{y}=0$

$\Gamma_{3}: u_{x}=0, w_{x}=0$

$\Gamma_{4}:$ free

Fig. 1 A) Geometry and boundary conditions of the column of soil; Loading of B) the dynamic consolidation and C) large deformation consolidation problems.

\subsection{Dynamic consolidation of a soil column}

The dynamic consolidation of a soil column is studied using the geometry given in Fig. 1.A. The column is loaded at the top boundary, $\Gamma_{4}$, by a harmonic surface loading, $P_{\max } \cos (\omega t)$, see Fig. 1.B, where the angular frequency $\omega$ is defined as $2 \pi / T$. This problem was first analytically solved by Zienkiewicz et al. [44] in 1980s, and more recently by Navas et al. using either implicit Eulerian method [29] or explicit Lagrangian schemes [28] employing LME shape functions.

The soil behavior studied in dependent on the solid skeleton properties, the permeability and the frequency of the harmonic load. Three zones, defined in Fig. 2 are characterized by the values of $\Pi_{1}$ and $\Pi_{2}$, which are defined as follows:

$\Pi_{1}=\frac{k V_{c}^{2}}{g \frac{\rho_{f}}{\rho} \omega H_{T}^{2}}=\frac{k \omega}{g \frac{\rho_{f}}{\rho} \Pi_{2}}, \quad \Pi_{2}=\frac{\omega^{2} H_{T}^{2}}{V_{c}^{2}}$

where $H_{T}$ is the column height, $V_{c}$ is the $p$-wave velocity calculated as:

$V_{c}=\sqrt{\left(D+\frac{K_{w}}{n}\right) \frac{1}{\rho}}$, where $D=\frac{2 G(1-\nu)}{1-2 \nu}$.

Zone I is characterized as slow phenomenon where both solid and fluid accelerations can be neglected; Zone II is typical of moderate speed behavior, where only the fluid phase inertia is negligible; in Zone III, however, inertial contributions from both solid and fluid phases are significant and cannot be neglected. The four different points studied herein are shown in Fig. 2, where 
Table 3 Material parameters of the dynamic consolidation problem

\begin{tabular}{llll}
\hline$G[\mathrm{MPa}]$ & 312.5 & $K_{w}[\mathrm{MPa}]$ & $10^{4}$ \\
\hline$\nu$ & 0.2 & $K_{s}[\mathrm{MPa}]$ & $10^{34}$ \\
\hline$n$ & 0.333 & $\rho_{w}\left[\mathrm{~kg} / \mathrm{m}^{3}\right]$ & 1000 \\
\hline$V_{c}[\mathrm{~m} / \mathrm{s}]$ & 3205 & $\rho_{s}\left[\mathrm{~kg} / \mathrm{m}^{3}\right]$ & 3003 \\
\hline
\end{tabular}

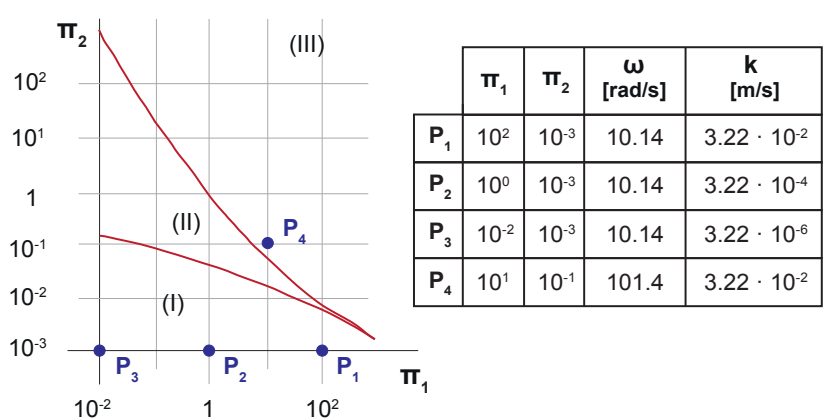

Zone (I) - Slow phenomena: ü and $\ddot{w}$ can be neglected

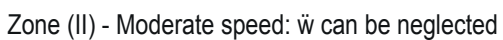

Zone (III) - Fast phenomena: only full Biot eq. valid

Fig. 2 Three zones that characterize the soil behavior in the $\Pi_{1}-\Pi_{2}$ space (See [44]), and tabulated parameter values for the different points to be studied.

the loading frequency, $\omega$, and the soil permeability, $k$, are also listed.

In order to evaluate the performance of the current implicit methodology, the maximum envelope of the dimensionless pore pressure along the column is depicted in Fig. 3. This figure shows an excellent agreement between the solutions given by the semi-analytical solution proposed by Zienkiewicz and coworkers [44], the explicit Lagrangian [28] scheme, and the one obtained with the present implicit Lagrangian methodology. The solution given by the current methodology presents more stability than the explicit one, even for the point $\mathrm{P}_{4}$, which lies in the Zone III.

\subsection{Large deformation consolidation of a soil column}

In order to validate the developed methodology against large deformation problems, the problem proposed by y Li, Borja and Regueiro [21] is studied. The geometry of the soil is the same as the previous example, see Fig. 1.A, whereas the load history is given in Fig. 1.C, where $t_{0}=0.05 \mathrm{~s}, P_{\max }=8 \mathrm{MPa}$. Parameters of the soil skeleton are provided in Tab. 4. The Neo-Hookean material model described in Section 4.1 is employed in this case since is more suitable to simulate the reduction

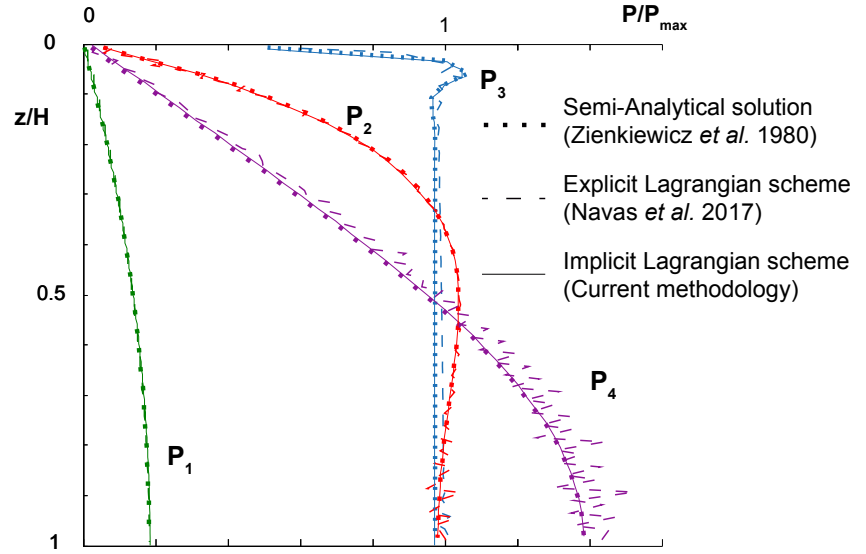

Fig. 3 Maximum envelopes of the isochrones of the pore pressure for points $P_{1}$ to $P_{4}$ solved through three different numerical schemes.

Table 4 Material parameters of the dynamic consolidation problem

\begin{tabular}{|ll|ll|}
\hline$\lambda[\mathrm{MPa}]$ & 29 & $K_{w}[\mathrm{MPa}]$ & $2.2 \cdot 10^{4}$ \\
\hline$G[\mathrm{MPa}]$ & 7 & $K_{s}[\mathrm{MPa}]$ & $10^{34}$ \\
\hline$n$ & 0.42 & $\rho_{w}\left[\mathrm{~kg} / \mathrm{m}^{3}\right]$ & 1000 \\
\hline$k[\mathrm{~m} / \mathrm{s}]$ & 0.1 & $\rho_{s}\left[\mathrm{~kg} / \mathrm{m}^{3}\right]$ & 2700 \\
\hline
\end{tabular}

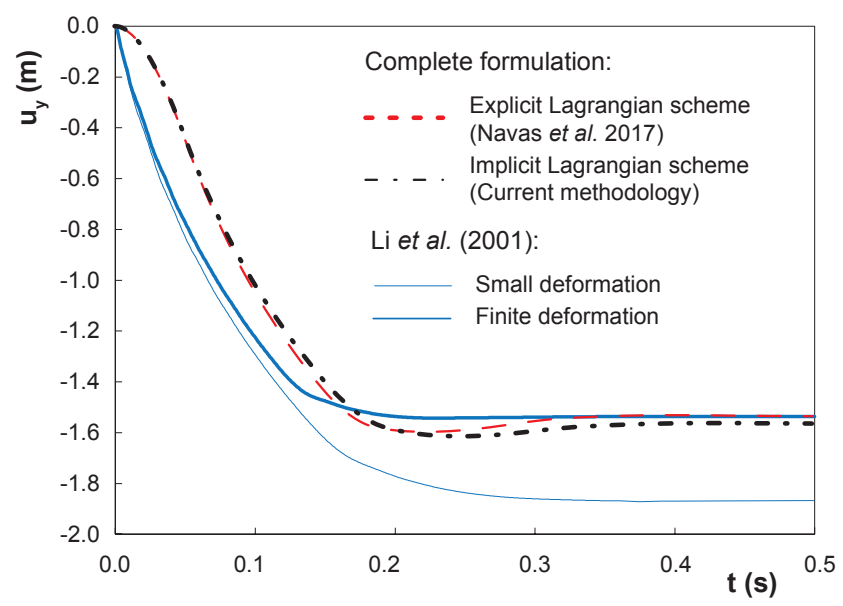

Fig. 4 Comparison between the settlement obtained by Li et al., the explicit solution and with the proposed methodology for the large deformation consolidation.

of the pores volume, i.e. the compaction, which leads to soil hardening of the soil with less settlement. The obtained solution is compared against those of Li, Borja and Regueiro [21] and Navas et al. [28] in Fig. 4. Quite similar settlement histories are obtained by the implicit and explicit Lagrangian schemes based on the $u-w$ formulation, in particular, the dynamic branch around $0.3 \mathrm{~s}$ are captured. By contrast, the solution based on 


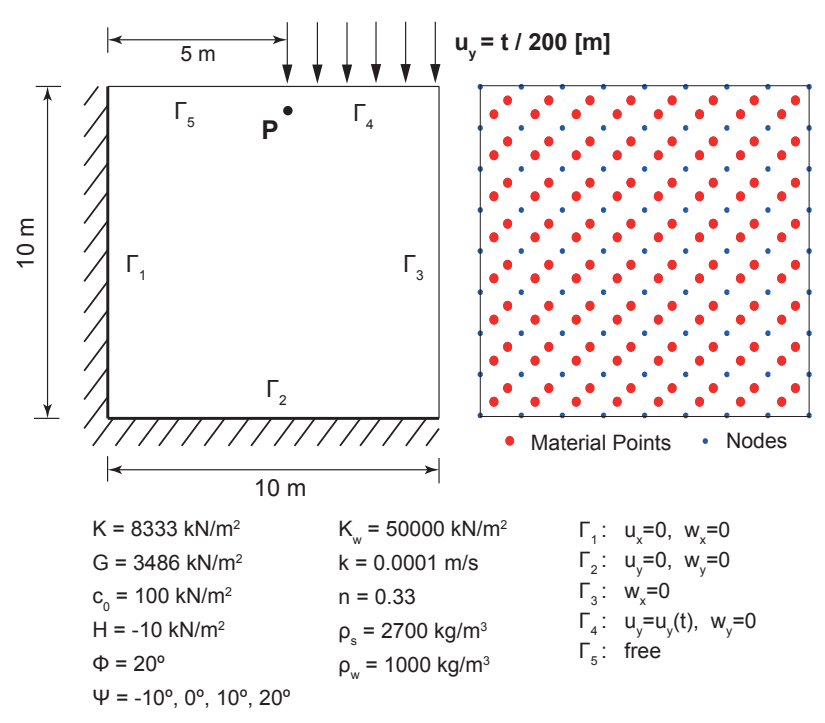

Fig. 5 Geometry, spatial discretization, material parameters and boundary conditions of a square domain of water saturated porous material.

the $u-p_{w}$ formulation by Li, Borja and Regueiro [21], the dynamic phenomenon has been smoothed out, since no acceleration terms were taken into account.

\subsection{Shear band formation in a square domain of} water saturated soil

The last validation is concerned with the formation of a shear band within a representative square domain of water saturated soil. The top right half of the domain is loaded by a rigid footing. The same problem was previously studied by Sanavia et al. $[33,34]$ in quasi-static conditions and [28] in dynamic conditions. The geometry and material properties are those shown in Fig. 5. A displacement of one meter is gradually imposed with a velocity of $5 \mathrm{~mm} / \mathrm{s}$ over a duration of $200 \mathrm{~s}$. The spatial discretization is also seen Fig. 5. It consists of 128 material points and 81 nodes. As it can be seen in the figure, no finer discretization has been assumed in the area where the shear band is expected to be found during the computation.

The distributions of the equivalent plastic strain and the pore pressure at $200 \mathrm{~s}$ for four different dilatancy angles are depicted in Fig. 6 and Fig. 7 respectively. No significant variations of the equivalent plastic strain are perceived for positive dilantancy angles (dilatant material), whereas large plastic strain is obtained for the negative one (contractive material). In addition, decreased shear band slopes are observed for smaller dilatancy angles. From Fig. 7, the effect of the plastic dilatancy (contractancy) is evidenced by the negative (positive) pore pressure within the shear band
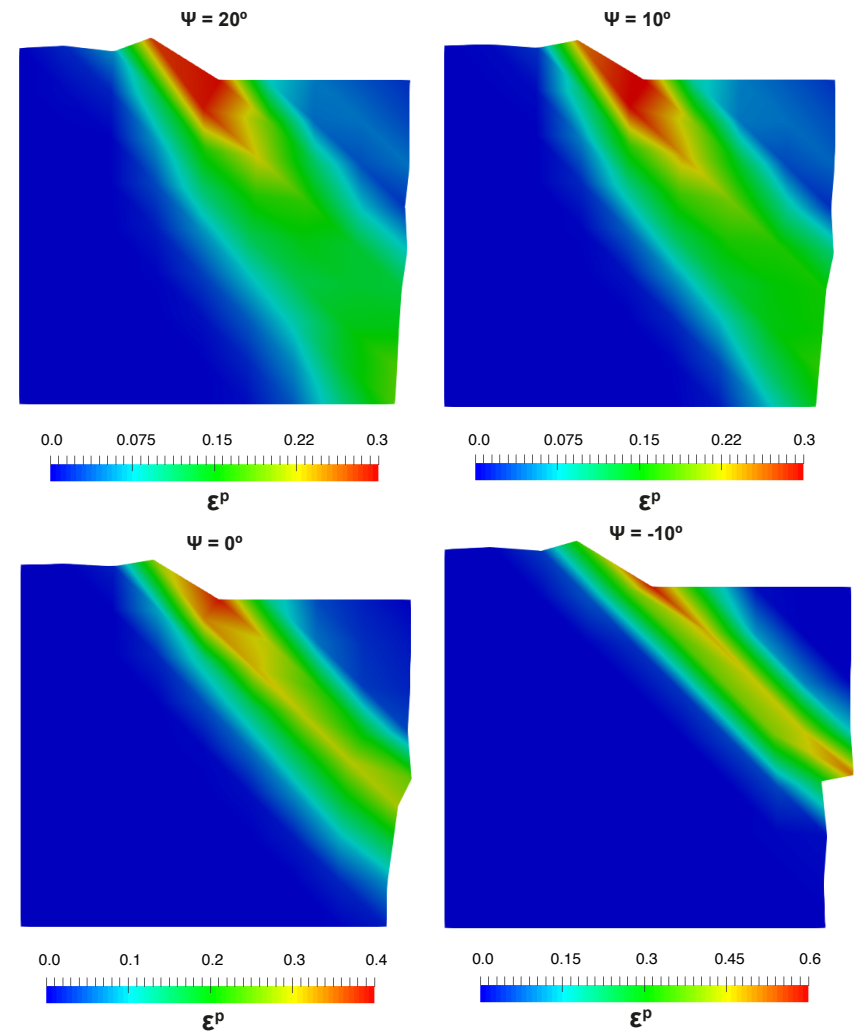

Fig. 6 Equivalent plastic strain spatial distribution at the final of the simulation for the four dilatancy angles.

zone, meanwhile in the case of zero dilatancy angle no marked pore pressure variation is observed within the shear band zone. Despite the coarse spatial discretization excellent results can be observed, mainly in the shear band zone.

Moreover, in the case of $\psi=20^{\circ}$, it can be noted in Fig. 8 that the negative water pressure within the shear band is smaller than the cavitation pressure at ambient temperature (-98986 Pa), indicating the occurrence of cavitation, as experimentally observed in [25]. This phenomenon should be modeled by extending the formulation of this paper to unsaturated conditions and adding the water vapor phase, e.g. as in [14].

In order to study the evolution of the principal results of the problem, the histories of the pore pressure and equivalent plastic strain in a material point close to the shear band ( $\mathrm{P}$, see Fig.5) have been extracted and depicted in Figs. 9 and 10 respectively. The evolution of the pore pressure shows an increase of when contractive material is employed meanwhile a reduction occurs for positive dilatancy angles, as expected. The higher plastic strain values for contractive materials is seen in Fig. 10. The evolution of the reaction forces against the footing (Fig. 11) also provides interesting information. Once the material plastifies, the 0 

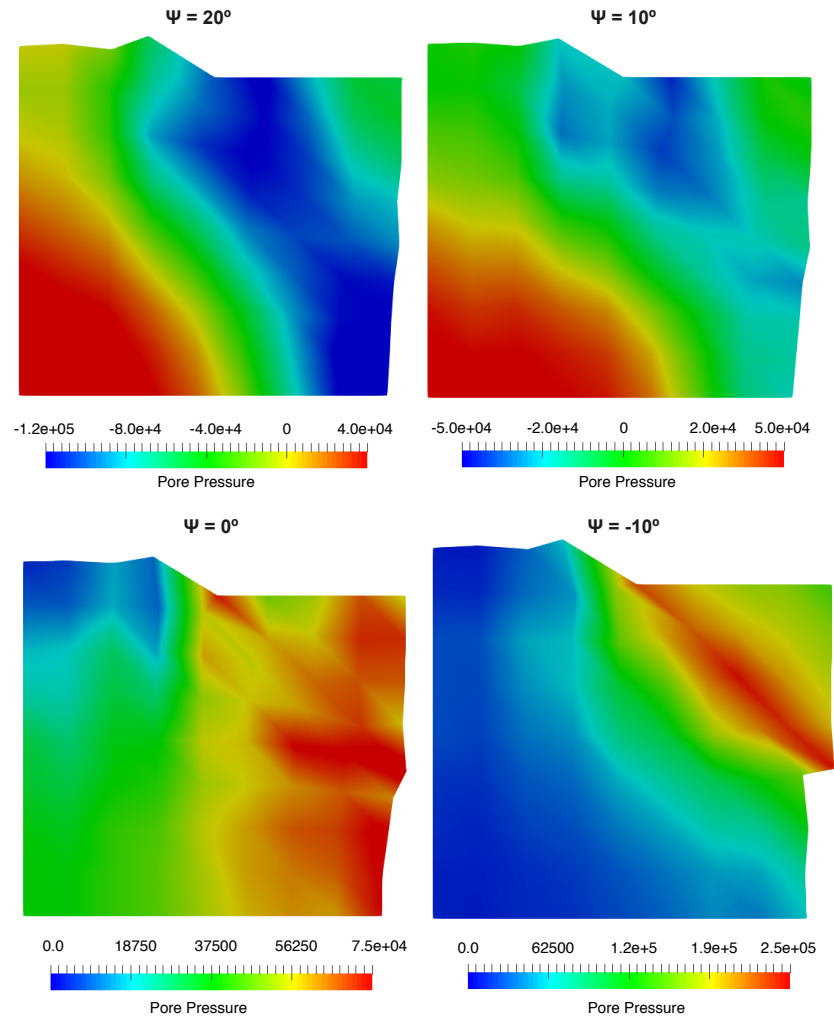

Fig. 7 Pore pressure distribution (in $\mathrm{Pa}$ ) at the final of the simulation for the four dilatancy angles.

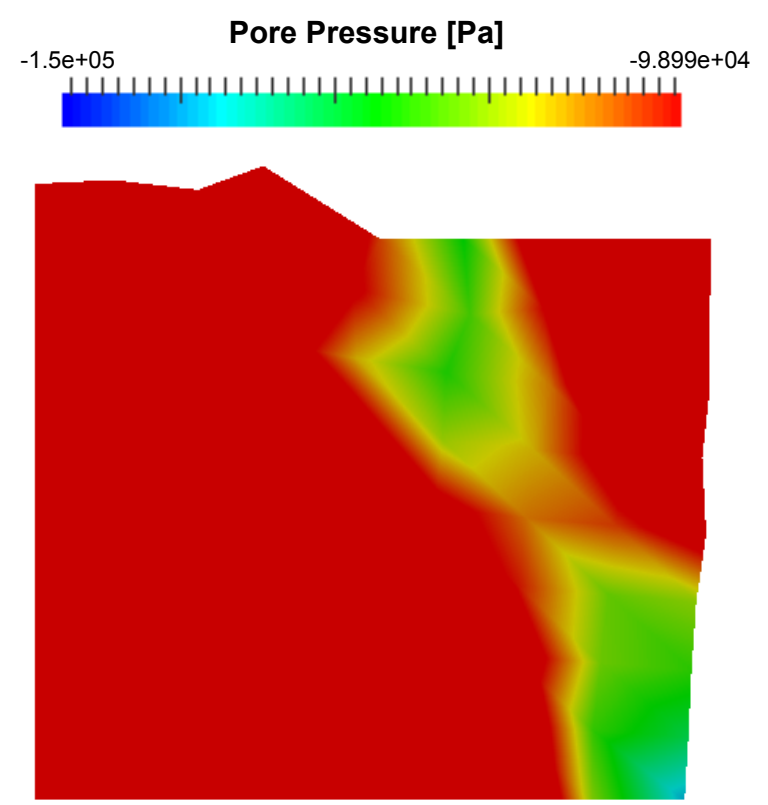

Fig. 8 Pore pressure distribution (in Pa) below the cavitation value $(98.99 \mathrm{kPa})$ for dilatancy equal to $20^{\circ}$ at the final of the simulation.

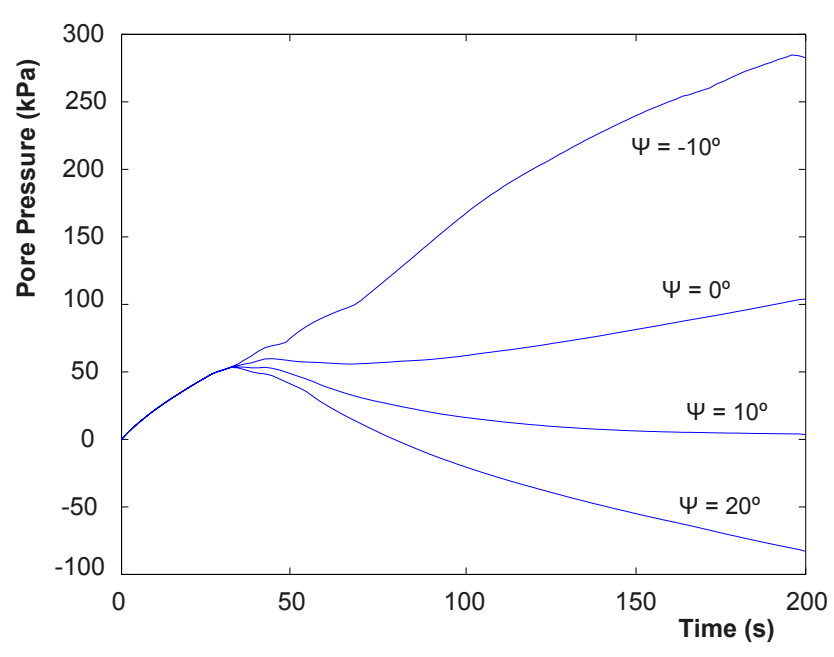

Fig. 9 Evolution of the pore pressure along the time in the point $P$.

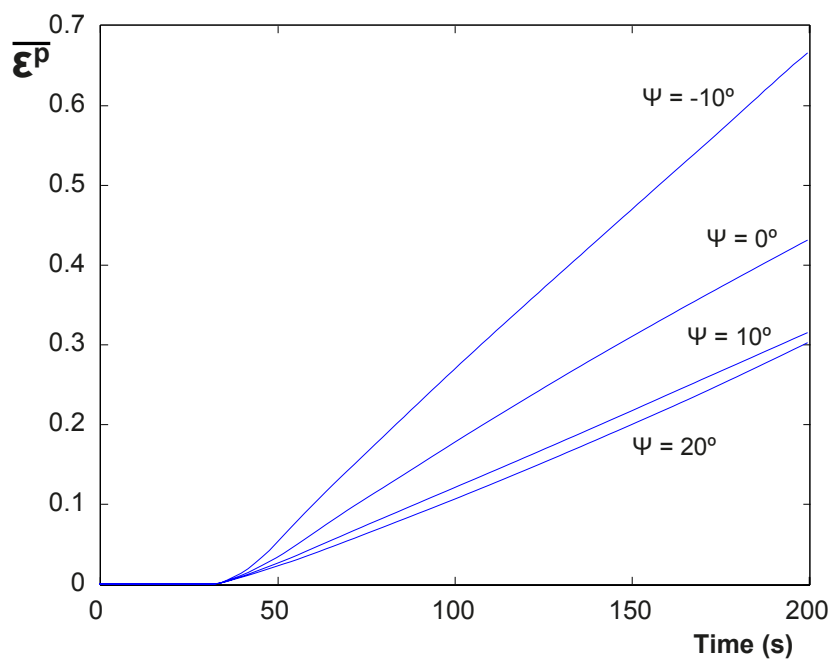

Fig. 10 Evolution of the equivalent plastic strain along the time in the point $\mathrm{P}$.

dilatancy angle material keeps the reaction constant, dilatant materials obtain a post-peak hardening while the contractive material feels the loss of the resistance which explains the occurrence of larger plastic strains and can be interpreted as the fracture of the soil.

The aspects of the regularization properties of the $u-w$ multiphase model at localization are not analyzed in this paper and will deserve further studies. For the $u-p_{w}$ model, the interested reader can see [36] and [8], while its internal length scale $l_{w}$ is presented in [42]. 


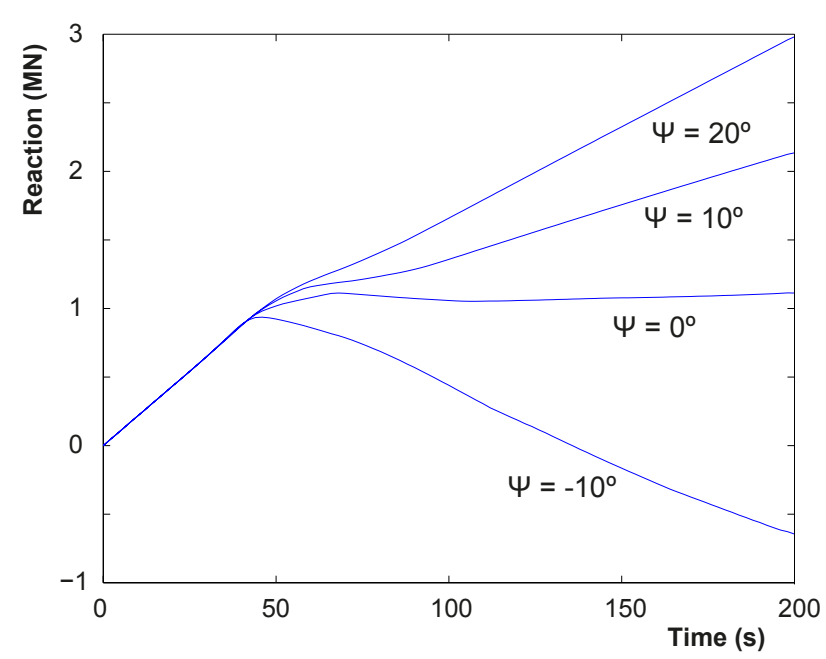

Fig. 11 Reaction forces of the soil for the different dilatancy angles.

\section{Application to an embankment loaded by a horizontal, harmonic, gravitational acceleration}

As mentioned before, the main advantages of the $u-w$ formulation in comparison to a $u-p_{w}$ one lie in its better suitability to accurately reproduce the soil behavior when high frequency dynamic loadings are involved. In this Section, we apply the developed implicit Lagrangian scheme to a realistic embankment loaded by a horizontal sinusoidal acceleration, representing the action of an earthquake with an amplitude of $g / 2$, and a frequency of $1 \mathrm{~Hz}$, i.e.

$$
a=\frac{g}{2} \sin (2 \pi f t)
$$

The geometry and material properties of the DruckerPrager soil are given in Fig. 12. The boundary conditions of the solid and fluid phases are also depicted in the figure, where the foundation borders $\left(\Gamma_{1}, \Gamma_{2}\right.$, and $\left.\Gamma_{3}\right)$ are impermeable. Two dilatancy angles, $5^{\circ}$ and $-3^{\circ}$, are adopted for this study. Unlike the previous examples, the gravity is necessarily considered here as initial stresses throughout the whose domain before the earthquake occurs are required to start the time integration. Consequently, an initial state was computed until an steady state of the pore pressure was achieved, and after that, the dynamic acceleration was applied. The seismic load is applied for a duration of $20 \mathrm{~s}$ (or until soil failure) as horizontal acceleration to all the nodes of the domain. Initial pore pressure conditions are hydrostatic, as shown in Fig.13. The distributions of the equivalent plastic strain at different times are illustrated in Fig. 14 and Fig. 15 for dilatancy angles of $5^{\circ}$ and $-3^{\circ}$ respectively. Note that for the former

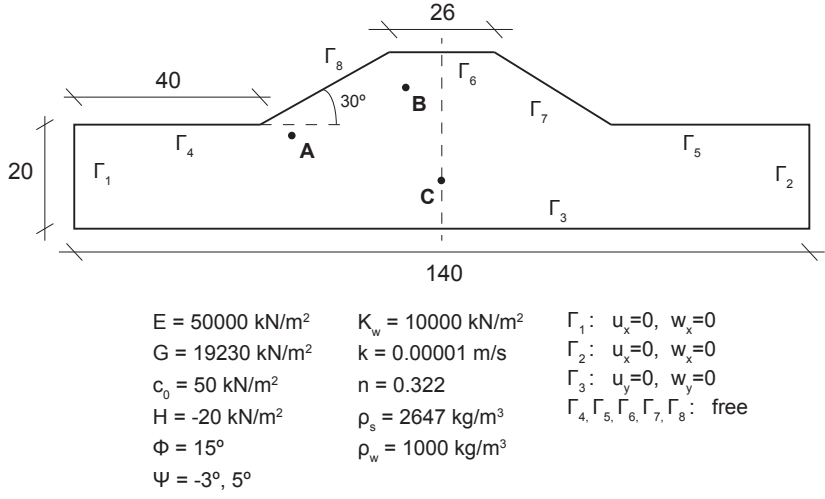

Fig. 12 Geometry (in m), material parameters and boundary conditions of the embankment problem.

\section{Pore Pressure}
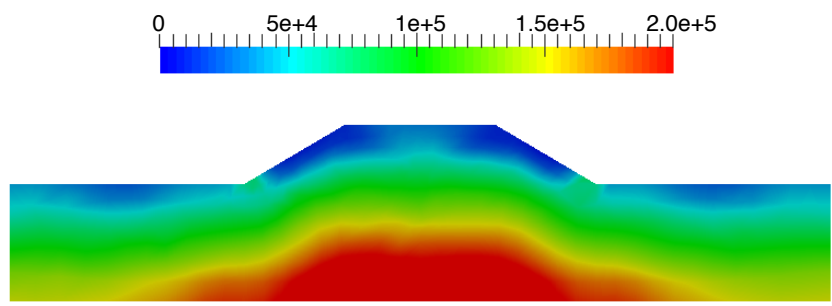

Fig. 13 Initial pore pressure conditions.

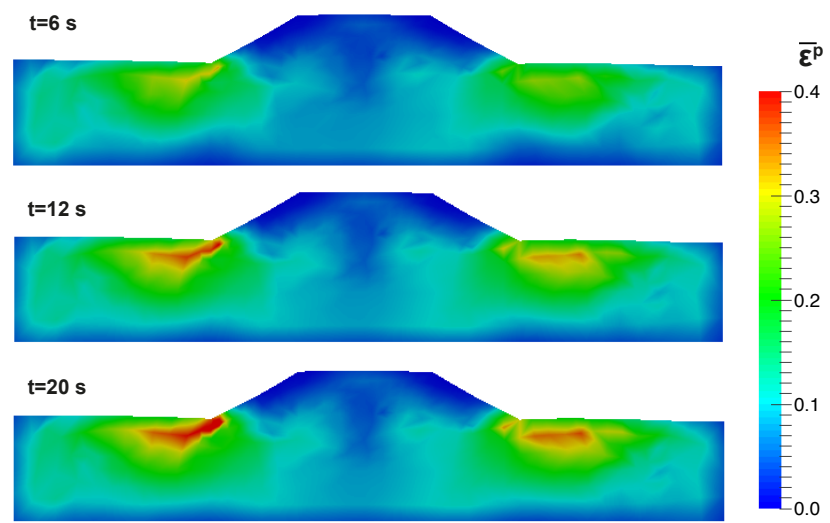

Fig. 14 Distribution of the equivalent plastic strain in the soil at 6,12 and $20 \mathrm{~s}, \psi=5^{\circ}$. (The displacements have been amplified by a factor of two)

(dilantant soil), the maximum plastic strains are concentrated around the area where the embankment intercepts the foundation, whereas global failure which involves particularly the foundation is observed for the latter (contractive soil). In addition, for the dilatancy angle of $-3^{\circ}$, since soil breakage occurred around $12 \mathrm{~s}$, no further calculations were carried out.

In order to gain more insights into the failure process, three points located around the expected failure 


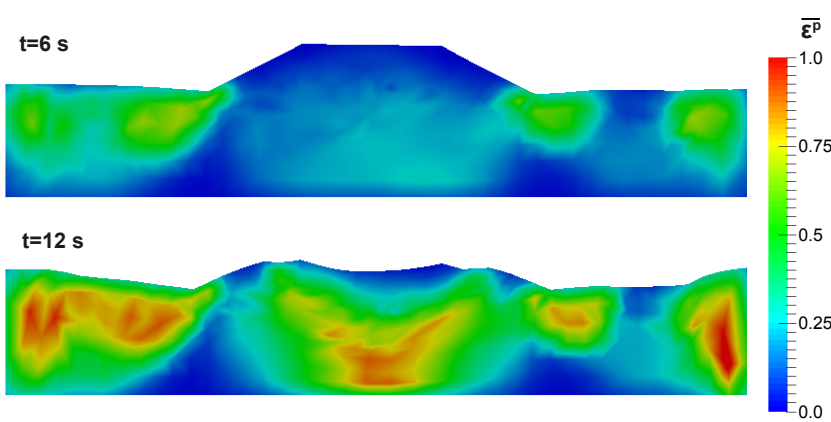

Fig. 15 Distribution of the equivalent plastic strain in the soil at $6,12 \mathrm{~s}, \psi=-3^{\circ}$. (The displacements have been amplified by a factor of two)

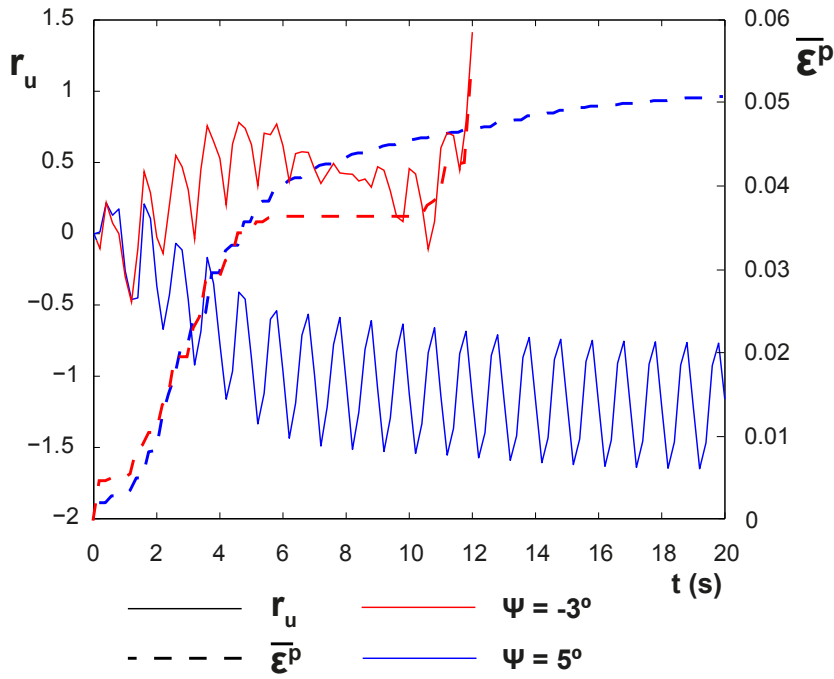

Fig. 16 Evolution of $r_{u}$ and $\overline{\varepsilon^{p}}$ at point A (see Fig. 12).

zone are chosen (A, B and C), see Fig. 12. Besides the equivalent plastic strain, the liquefaction ratio, $r_{u}$, is also employed. It is defined as the overpressure increment of water with respect to the initial pore pressure, normalized by the the initial average effective stress, $p_{0}^{\prime}$, i.e.

$r_{u}=\frac{p_{w}-p_{w_{0}}}{p_{0}^{\prime}}$.

This ratio measures the state of pressures in the saturated soil under an earthquake load, having in mind that a value of 1.0 points to the liquefaction of the points where this is reached.

The evolutions of both the liquefaction ratio (continuous lines) and the equivalent plastic strain (discontinuous lines) are plotted in Figs. 16, 17 and 18 for the points A, B and C respectively. The results for dilatancy angles of $5^{\circ}$ are shown in blue color, whereas those for $-3^{\circ}$ are represented in red. Note that for dilatant soil, the evolution of the liquefaction ratio reaches a harmonic steady state of the same frequency $(1 \mathrm{~Hz})$, at

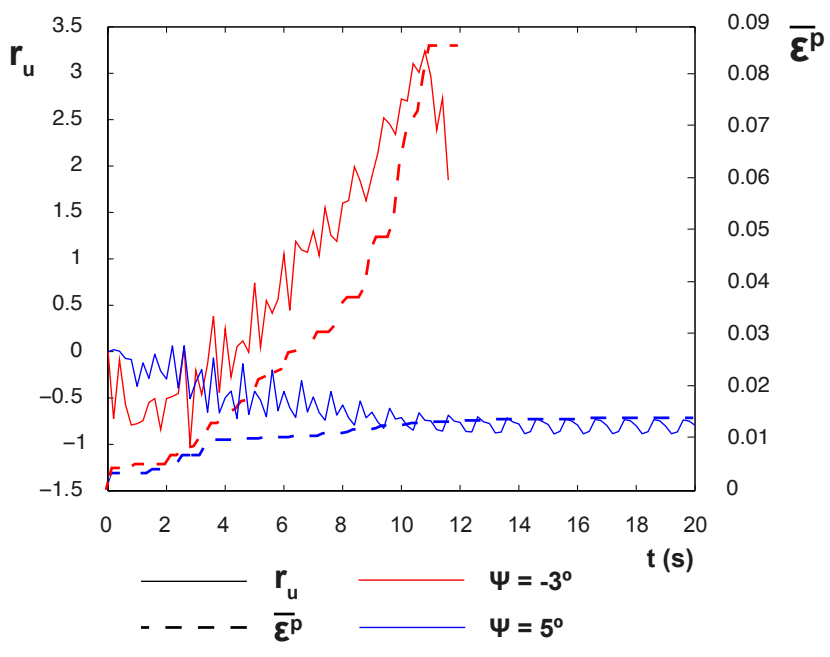

Fig. 17 Evolution of $r_{u}$ and $\overline{\varepsilon^{p}}$ at point B (see Fig. 12).

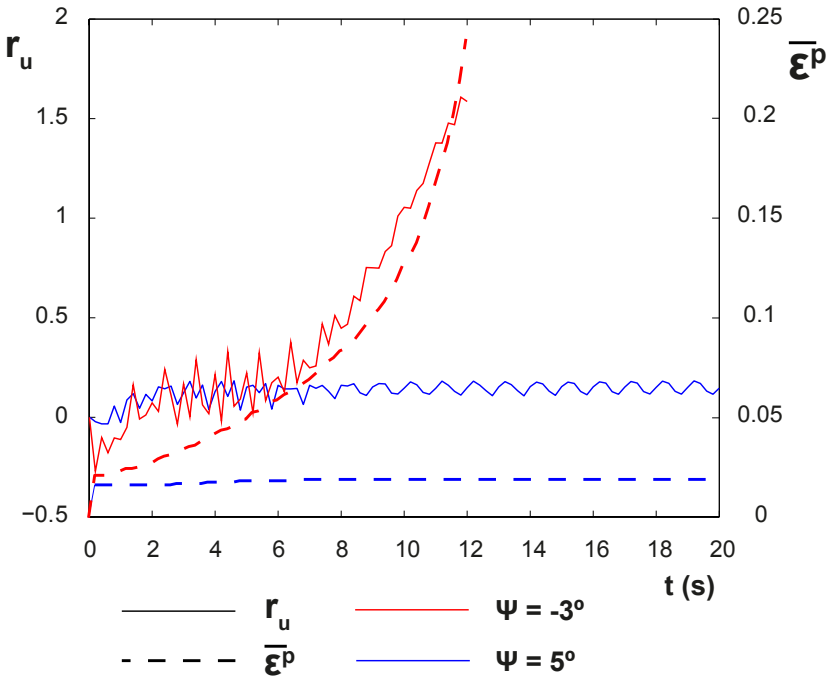

Fig. 18 Evolution of $r_{u}$ and $\overline{\varepsilon^{p}}$ at point C (see Fig. 12).

the same time, a near-constant equivalent plastic strain is attained at all three points and shows that liquefaction cannot occur as expected for dilatant materials. By contrast, an abrupt increase of both the liquefaction ratio and the equivalent plastic strain at $12 \mathrm{~s}$ causing soil failure is observed for the dilatancy angle of $-3^{\circ}$.

\section{Conclusions}

The main goal of this paper is the design of a methodology capable to model the full dynamic behavior of a saturated soil under large deformation conditions. The application of an implicit time integration scheme in the resolution of the motion equations implies a strong mathematical formulation, since it is necessary to lin- 
earize the derivatives of the linear momentum equations of both solid and fluid phases (see Appendix A).

This research supposes the first development of the linearization of the $u-w$ formulation, useful to solve large deformation problems with several computational methods. Despite the higher mathematical effort required by this formulation, as well as the more laborious implementation compared to the explicit one, the range of applicability of this implicit scheme is wider than the explicit one, the formulation of which is much simpler than the one proposed in this paper. However explicit formulations are only suitable for high loading rates problems, as the time intervals required for convergence in such methodologies are prohibitive in terms of computational efforts. On the other hand, in the same range of problems, with similar spatial discretizations, the implicit approach presents higher stability, which is crucial when the deformations become higher.

The derivation of the motion equations is successfully reached and subsequently validated against reference examples through a meshfree framework, LME, which is more suitable for large deformation problems. The performance of three different aspects were assessed in this research: the behavior under high frequency loads; the results of large settlement in a consolidation problem; and the applicability of the methodology for different constitutive materials. The main conclusion derived from the obtained results is the better stability achieved in comparison with the explicit solution previously reported by [28]. This fact may be evidenced by observing the evolution of the pore pressures along time for the different tests carried out in this paper: the convergence is smoother and more stable. In addition, the results are in good agreement with the results of the referenced research, suggesting a good accuracy of the methodology as well. The spatial distribution of the pore pressure also indicates the strength of the methodology.

Although the validation tests offer excellent results, they only lie on the academic field. Thus, the employment of the proposed method in the study of the behavior of a realistic embankment loaded by an earthquake helps us to complete this study with a more realistic dynamic problem. In this case, the liquefaction is the main subject of research since it may be a measurement of the failure of the material. This behavior has been studied in both dilatant and contractive material, the last being more in our interest since the typical increase of the pore pressure, which occurs in this type of soils, may lead to the rise of the liquefaction ratio, as it is shown in this paper. Therefore, we can conclude that the proposed methodology is also capable to capture liquefaction failures in saturated, granular soils when dynamically loaded.

Finally, once the method is tested, future research may employ it with different constitutive models in order to simulate different soils, depending on the different material properties. Also, it is required the assessment of the usage of different time integration schemes (see [29] and [1]) in order to improve the performance of the proposed methodology.

Acknowledgements The financial support to develop this research from the Ministerio de Ciencia e Innovación, under Grant Numbers, BIA2012-31678 and BIA2015-68678-C2-1-R, and the Consejería de Educación, Cultura y Deportes de la Junta de Comunidades de Castilla-La Mancha, Fondo Europeo de Desarrollo Regional, under Grant No. PEII-2014016-P, Spain, is greatly appreciated. The first author also acknowledges the fellowship BES2013-0639 as well as the fellowship EEBB-I-17-12624 which supported him on his stay in DICEA, University of Padova (Italy). The second author also would like to thank the University of Padova, Italy (research grant DOR1725272/17). 


\section{A Appendix: Consistent Linearization}

As the linearization is referred to the undeformed domain, $B_{0}$, since it is time independent, it is necessary to move Eqs. (31$32)$ to the reference configuration. From the transport theorems we know that $d v=J d V$ and $d s=J \boldsymbol{F}^{-T} d S$ and the Piola transformation states that $\operatorname{Div}(\boldsymbol{u})=J \operatorname{div}(\boldsymbol{u})$ (See [24, 35] for more information). Starting from these points, the equations to be linearized yield

$-\alpha_{7} \int_{B_{0}} \boldsymbol{\tau}^{\prime}: \operatorname{grad}(\delta \boldsymbol{u}) d V-\alpha_{7} \int_{B_{0}} Q \operatorname{Div}(\boldsymbol{u}) \operatorname{div}(\delta \boldsymbol{u}) d V$

$-\alpha_{7} \int_{B_{0}} Q \operatorname{Div}(\boldsymbol{w}) \operatorname{div}(\delta \boldsymbol{u}) d V-\alpha_{1} \int_{B_{0}}\left[\rho_{0} \boldsymbol{u}+J \rho_{w} \boldsymbol{w}\right] \cdot \delta \boldsymbol{u} d V$

$+\alpha_{8} \int_{B_{0}} \rho_{0} \boldsymbol{g} \cdot \delta \boldsymbol{u} d V+\alpha_{8} \int_{\delta B_{0}} \overline{\boldsymbol{T}} \cdot \delta \boldsymbol{u} d S=0$

$-\alpha_{7} \int_{B_{0}} Q \operatorname{Div}(\boldsymbol{u}) \operatorname{div}(\delta \boldsymbol{w}) d V-\alpha_{7} \int_{B_{0}} Q \operatorname{Div}(\boldsymbol{w}) \operatorname{div}(\delta \boldsymbol{w}) d V$

$-\alpha_{4} \int_{B_{0}} \frac{J \mu_{w}}{k} \boldsymbol{w} \cdot \delta \boldsymbol{w} d V-\alpha_{1} \int_{B_{0}} \frac{J \rho_{w}}{n} \boldsymbol{w} \cdot \delta \boldsymbol{w} d V$

$-\alpha_{1} \int_{B_{0}} J \rho_{w} \boldsymbol{u} \cdot \delta \boldsymbol{w} d V+\alpha_{8} \int_{B_{0}} J \rho_{w} \boldsymbol{g} \cdot \delta \boldsymbol{w} d V$

$-\alpha_{8} \int_{\delta B_{0}} \overline{\boldsymbol{T}}_{w} \cdot \delta \boldsymbol{w} d S=0$,

where $\boldsymbol{\tau}^{\prime}$ is the effective Kirchhoff stress tensor and $\overline{\boldsymbol{T}}$ and $\overline{\boldsymbol{T}}_{w}$ are respectively the traction vectors of solid and fluid phases computed respect the undeformed configuration.

Before linearizing the different terms of the target equation, the linearization of some useful terms is carried out against $\Delta \boldsymbol{u}$ :

$D_{u}[J]=J \operatorname{div}(\Delta \boldsymbol{u})$

(From [16]) Also the linearization of $n$ will be useful for the derivation of other quantities:

$$
\begin{aligned}
D_{u}[n] & =D_{u}\left[1-\frac{1-n_{0}}{J}\right]=-\left(1-n_{0}\right) \frac{-1}{J^{2}} D_{u}[J] \\
& =\frac{1-n_{0}}{J} \operatorname{div}(\Delta \boldsymbol{u})=(1-n) \operatorname{div}(\Delta \boldsymbol{u})
\end{aligned}
$$

From tensor analysis [15] we determine that:

$$
\begin{aligned}
D_{u}[\operatorname{grad}(\boldsymbol{u})] & =D_{u}\left[\operatorname{Grad}(\boldsymbol{u}) \boldsymbol{F}^{-1}\right] \\
& =\operatorname{Grad}\left(D_{u}[\boldsymbol{u}]\right) \boldsymbol{F}^{-1}+\operatorname{Grad}(\delta \boldsymbol{u}) D_{u}\left[\boldsymbol{F}^{-1}\right] \\
& =\operatorname{Grad}(\Delta \boldsymbol{u}) \boldsymbol{F}^{-1}-\operatorname{Grad}(\boldsymbol{u}) \boldsymbol{F}^{-1} \operatorname{grad}(\Delta \boldsymbol{u}) \\
& =\operatorname{grad}(\Delta \boldsymbol{u})-\operatorname{grad}(\boldsymbol{u}) \operatorname{grad}(\Delta \boldsymbol{u})
\end{aligned}
$$

$$
\begin{aligned}
D_{u}[\operatorname{div}(\delta \boldsymbol{u})] & =\boldsymbol{I}: D_{u}[\operatorname{grad}(\delta \boldsymbol{u})] \\
& =\boldsymbol{I}:\left[\underline{\operatorname{Grad}\left(D_{u}[\delta \boldsymbol{u}]\right) \boldsymbol{F}^{-1}}-\operatorname{grad}(\delta \boldsymbol{u}) \operatorname{grad}(\Delta \boldsymbol{u})\right] \\
& =-\operatorname{grad}(\delta \boldsymbol{u}): \operatorname{grad}^{T}(\Delta \boldsymbol{u}) \\
D_{u}[\operatorname{Div}(\boldsymbol{u})] & =D_{u}[\boldsymbol{I}: \operatorname{Grad}(\boldsymbol{u})]=\boldsymbol{I}: D_{u}[\operatorname{Grad}(\boldsymbol{u})] \\
& =\boldsymbol{I}: \operatorname{Grad}\left(D_{u}[\boldsymbol{u}]\right)=\boldsymbol{I}: \operatorname{Grad}(\Delta \boldsymbol{u})=\operatorname{Div}(\Delta \boldsymbol{u})
\end{aligned}
$$

So, the term we can see in the linear momentum balance equations is linearized as follows:

$$
\begin{aligned}
D_{u}[\operatorname{Div}(\boldsymbol{u}) \operatorname{div}(\delta \boldsymbol{u})]= & D_{u}[\operatorname{Div}(\boldsymbol{u})] \operatorname{div}(\delta \boldsymbol{u}) \\
& +\operatorname{Div}(\boldsymbol{u}) D_{u}[\operatorname{div}(\delta \boldsymbol{u})] \\
= & \operatorname{grad}(\delta \boldsymbol{u}):[\operatorname{Div}(\Delta \boldsymbol{u}) \boldsymbol{I} \\
& \left.-\operatorname{Div}(\boldsymbol{u}) \operatorname{grad}^{T}(\Delta \boldsymbol{u})\right]
\end{aligned}
$$

Other important linearizations can be derived from Eq.A.4

$$
D_{u}[k]=(1-n) \frac{\partial k}{\partial n} \operatorname{div}(\Delta \boldsymbol{u})
$$

(See also [35])

$$
\begin{aligned}
D_{u}\left[J \frac{\mu}{k}\right] & =\frac{\mu}{k} J \operatorname{div}(\Delta \boldsymbol{u})-J \frac{\mu}{k^{2}} D_{u}[k] \\
& =J \frac{\mu}{k}\left[1-\frac{1-n}{k} \frac{\partial k}{\partial n}\right] \operatorname{div}(\Delta \boldsymbol{u}) \\
D_{u}[Q]= & D_{u}\left[\frac{K_{w}}{n}\right]=K_{w} \frac{\partial}{\partial n}\left[\frac{1}{n}\right] \frac{\partial n}{\partial \boldsymbol{u}}=-\frac{K_{w}}{n^{2}} D_{u}[n] \\
= & \frac{K_{w}}{n^{2}}(1-n) \operatorname{div}(\Delta \boldsymbol{u}) \\
D_{u}\left[J \frac{\rho_{w}}{n}\right] & =\frac{\rho_{w}}{n} J \operatorname{div}(\Delta \boldsymbol{u})-J \frac{\rho_{w}}{n^{2}}(1-n) \operatorname{div}(\Delta \boldsymbol{u}) \\
& =J \frac{\rho_{w}}{n} \frac{2 n-1}{n} \operatorname{div}(\Delta \boldsymbol{u})
\end{aligned}
$$

As the reference density is defined as

$\rho_{0}=J \rho=J n \rho_{w}+J(1-n) \rho_{s}$

$$
=J \rho_{w}-\left(1-n_{0}\right) \rho_{w}+\left(1-n_{0}\right) \rho_{s},
$$

the linearization of the density yields:

$$
D_{u}\left[\rho_{0}\right]=D_{u}\left[J \rho_{w}\right]=J \rho_{w} \operatorname{div}(\Delta \boldsymbol{u})
$$

The linearization will be stated for the weak form with respect to the reference configuration. Hereinafter the linearization of the terms that upon the deformation field are presented. All other terms will take part of the Newton scheme in the sense that it presented in Section 3. In the following equations superscripts represent the different terms of both Linear Momentum Balance equation of mixture and fluid phases respectively.

$-D G_{L M S} \cdot \Delta \boldsymbol{u}$ :

$$
\begin{aligned}
D G_{L M S} \stackrel{1}{M} \cdot \Delta \boldsymbol{u}= & D_{u}\left[\boldsymbol{\tau}^{\prime}: \operatorname{grad}(\delta \boldsymbol{u})\right] \\
= & \operatorname{grad}(\Delta \boldsymbol{u}) \boldsymbol{\tau}^{\prime}: \operatorname{grad}(\delta \boldsymbol{u}) \\
& +J \operatorname{grad}(\Delta \boldsymbol{u}): \boldsymbol{C}^{e p}: \operatorname{grad}(\delta \boldsymbol{u})
\end{aligned}
$$

where $\boldsymbol{C}^{e p}$ is the material elasto-plastic constitutive tangent operator. This linearization is widely developed in literature [40].

$$
\begin{aligned}
D G_{L} \stackrel{M}{S}_{S} \cdot \Delta \boldsymbol{u}= & D_{u}[Q \operatorname{Div}(\boldsymbol{u}) \operatorname{div}(\delta \boldsymbol{u})] \\
= & D_{u}[Q] \operatorname{Div}(\boldsymbol{u}) \operatorname{div}(\delta \boldsymbol{u}) \\
& +Q D_{u}[\operatorname{Div}(\boldsymbol{u}) \operatorname{div}(\delta \boldsymbol{u})] \\
= & Q\left(-\frac{1-n}{n} \operatorname{div}(\Delta \boldsymbol{u}) \operatorname{Div}(\boldsymbol{u}) \operatorname{div}(\delta \boldsymbol{u})\right. \\
& \left.+\operatorname{grad}(\delta \boldsymbol{u}):\left[\operatorname{Div}(\Delta \boldsymbol{u}) \boldsymbol{I}-\operatorname{Div}(\boldsymbol{u}) \operatorname{grad}^{T}(\Delta \boldsymbol{u})\right]\right) \\
= & J Q \operatorname{grad}(\delta \boldsymbol{u}):(\operatorname{div}(\Delta \boldsymbol{u}) \boldsymbol{I} \\
& \left.-\operatorname{div}(\boldsymbol{u})\left[\operatorname{grad}^{T}(\Delta \boldsymbol{u})+\frac{1-n}{n} \operatorname{div}(\Delta \boldsymbol{u}) \boldsymbol{I}\right]\right)
\end{aligned}
$$




$$
\begin{aligned}
D G_{L M S}^{3} \cdot \Delta \boldsymbol{u}= & D_{u}[Q \operatorname{Div}(\boldsymbol{w}) \operatorname{div}(\delta \boldsymbol{u})] \\
= & D_{u}[Q] \operatorname{Div}(\boldsymbol{w}) \operatorname{div}(\delta \boldsymbol{u}) \\
& +Q D_{u}[\operatorname{Div}(\boldsymbol{w})] \operatorname{div}(\delta \boldsymbol{u}) \\
& +Q \operatorname{Div}(\boldsymbol{w}) D_{u}[\operatorname{div}(\delta \boldsymbol{u})] \\
= & -J Q \operatorname{grad}(\delta \boldsymbol{u}):\left(\operatorname { d i v } ( \boldsymbol { w } ) \left[\operatorname{grad}^{T}(\Delta \boldsymbol{u})\right.\right. \\
& \left.\left.+\frac{1-n}{n} \operatorname{div}(\Delta \boldsymbol{u}) \boldsymbol{I}\right]\right) \\
D G_{L M S} \stackrel{4}{M} \cdot \Delta \boldsymbol{u}= & D_{u}\left[\rho_{0} \boldsymbol{u}+J \rho_{w} \boldsymbol{w}\right] \cdot \delta \boldsymbol{u} \\
= & D_{u}\left[J \rho_{w}\right](\boldsymbol{u}+\boldsymbol{w}) \cdot \delta \boldsymbol{u}+J \rho D_{u}[\boldsymbol{u}] \cdot \delta \boldsymbol{u} \\
= & J\left[\rho \Delta \boldsymbol{u}+\rho_{w} \operatorname{div}(\Delta \boldsymbol{u})(\boldsymbol{u}+\boldsymbol{w})\right] \cdot \delta \boldsymbol{u}
\end{aligned}
$$

$-D G_{L M S} \cdot \Delta w$

$$
\begin{aligned}
D G_{L} \stackrel{2}{M S} \cdot \Delta \boldsymbol{w} & =D_{w}[Q \operatorname{Div}(\boldsymbol{u}) \operatorname{div}(\delta \boldsymbol{u})]=0 \\
D G_{L} \stackrel{3}{M S} \cdot \Delta \boldsymbol{w} & =D_{w}[Q \operatorname{Div}(\boldsymbol{w}) \operatorname{div}(\delta \boldsymbol{u})] \\
& =J Q \operatorname{grad}(\delta \boldsymbol{u}): \operatorname{div}(\Delta \boldsymbol{w}) \boldsymbol{I}
\end{aligned}
$$

$-D G_{L M W} \cdot \Delta \boldsymbol{u}:$

$$
\begin{aligned}
& D G_{L M W} \stackrel{1}{M} \cdot \Delta \boldsymbol{u}=D_{u}[Q \operatorname{div}(\boldsymbol{u}) \operatorname{div}(\delta \boldsymbol{w})] \\
& =J Q \operatorname{grad}(\delta \boldsymbol{w}):(\operatorname{div}(\Delta \boldsymbol{u}) \boldsymbol{I} \\
& \left.-\operatorname{div}(\boldsymbol{u})\left[\operatorname{grad}^{T}(\Delta \boldsymbol{u})+\frac{1-n}{n} \operatorname{div}(\Delta \boldsymbol{u}) \boldsymbol{I}\right]\right) \\
& D G_{L} \stackrel{2}{M} W \cdot \Delta \boldsymbol{u}=D_{u}[Q \operatorname{div}(\boldsymbol{w}) \operatorname{div} \delta \boldsymbol{w}] \\
& =-J Q \operatorname{grad}(\delta \boldsymbol{w}):\left(\operatorname { d i v } ( \boldsymbol { w } ) \left[\operatorname{grad}^{T}(\Delta \boldsymbol{u})\right.\right. \\
& \left.\left.+\frac{1-n}{n} \operatorname{div}(\Delta \boldsymbol{u}) \boldsymbol{I}\right]\right) \\
& D G_{L} \stackrel{3}{M W} \cdot \Delta \boldsymbol{u}=D_{u}\left[J \frac{\mu_{w}}{k} \boldsymbol{w} \cdot \delta \boldsymbol{w}\right] \\
& =D_{u}[J] \frac{\mu_{w}}{k} \boldsymbol{w} \cdot \delta \boldsymbol{w}+J D_{u}\left[\frac{\mu_{w}}{k}\right] \boldsymbol{w} \cdot \delta \boldsymbol{w} \\
& +\frac{J \mu_{w}}{k} D_{a}[\boldsymbol{w}\rceil \cdot \delta \boldsymbol{w} \\
& =J \frac{\mu_{w}}{k}\left[\operatorname{div}(\Delta \boldsymbol{u})\left(1-\frac{1-n}{k} \frac{\partial k}{\partial n}\right) \boldsymbol{w}\right] \cdot \delta \boldsymbol{w}
\end{aligned}
$$

$$
\begin{aligned}
D G_{L M W} \stackrel{4}{M}^{\prime} \cdot \Delta \boldsymbol{u}= & D_{u}\left[J \frac{\rho_{w}}{n} \boldsymbol{w} \cdot \delta \boldsymbol{w}\right] \\
= & D_{u}[J] \frac{\rho_{w}}{n} \boldsymbol{w} \cdot \delta \boldsymbol{w}+D_{u}\left[\frac{\rho_{w}}{n}\right] J \boldsymbol{w} \cdot \delta \boldsymbol{w} \\
& +\frac{J \rho_{w}}{n} D_{u}[\boldsymbol{w}\rceil \cdot \delta \boldsymbol{w} \\
= & \frac{J \rho_{w}}{n}\left[\frac{2 n-1}{n} \operatorname{div}(\Delta \boldsymbol{u}) \boldsymbol{w}\right] \cdot \delta \boldsymbol{w}
\end{aligned}
$$

$$
\begin{aligned}
D G_{L M W} \stackrel{5}{5} \cdot \Delta \boldsymbol{u} & =D_{u}\left[J \rho_{w} \boldsymbol{u} \cdot \delta \boldsymbol{w}\right] \\
& =\left[D_{u}[J] \rho_{w} \boldsymbol{u}+J \rho_{w} D_{u}[\boldsymbol{u}]\right] \cdot \delta \boldsymbol{w} \\
& =J \rho_{w}[\Delta \boldsymbol{u}-\operatorname{div}(\Delta \boldsymbol{u}) \boldsymbol{u}] \cdot \delta \boldsymbol{w}
\end{aligned}
$$

$$
\begin{aligned}
& -D G_{L M W} \cdot \Delta \boldsymbol{w}: \\
& D G_{L \stackrel{1}{M} W}^{\stackrel{1}{*}} \cdot \Delta \boldsymbol{w}=D_{w}[Q \operatorname{div}(\boldsymbol{u}) \operatorname{div}(\delta \boldsymbol{w})]=0 \\
& D G_{L} \stackrel{2}{M} W \cdot \Delta \boldsymbol{w}=D_{w}[Q \operatorname{div}(\boldsymbol{w}) \operatorname{div}(\delta \boldsymbol{w})] \\
& =J Q \operatorname{grad}(\delta \boldsymbol{w}): \operatorname{div}(\Delta \boldsymbol{w}) \boldsymbol{I}
\end{aligned}
$$

Finally, using the different terms carried out in the Eqs. (A.15A.27), the linearization of Eqs. (A.1-A.2) gives the following result:

$$
\begin{aligned}
& -\alpha_{7} \int_{B} \operatorname{grad}(\delta \boldsymbol{u}): \boldsymbol{c}^{e p}: \operatorname{grad}(\Delta \boldsymbol{u}) d v \\
& -\alpha_{7} \int_{B} \boldsymbol{\sigma}^{\prime}: \operatorname{grad}^{T}(\delta \boldsymbol{u}) \operatorname{grad}(\Delta \boldsymbol{u}) d v \\
& -\alpha_{7} \int_{B} \operatorname{grad}(\delta \boldsymbol{u}):(Q[\operatorname{div}(\Delta \boldsymbol{u})+\operatorname{div}(\Delta \boldsymbol{w})] \boldsymbol{I}) d v \\
& -\alpha_{7} \int_{B} \operatorname{grad}(\delta \boldsymbol{u}):\left(p_{w}\left[\operatorname{grad}^{T}(\Delta \boldsymbol{u})+\frac{1-n}{n} \operatorname{div}(\Delta \boldsymbol{u}) \boldsymbol{I}\right]\right) d v \\
& -\alpha_{1} \int_{B} \delta \boldsymbol{u} \cdot\left[\rho \Delta \boldsymbol{u}+\rho_{w} \Delta \boldsymbol{w}+\rho_{w} \operatorname{div}(\Delta \boldsymbol{u})(\boldsymbol{u}+\boldsymbol{w})\right] d v \\
& +\alpha_{8} \int_{B} \rho_{w} \delta \boldsymbol{u} \cdot \boldsymbol{g} \operatorname{div}(\Delta \boldsymbol{u}) d v
\end{aligned}
$$

$-\alpha_{7} \int_{B} \operatorname{grad}(\delta \boldsymbol{w}):(Q[\operatorname{div}(\Delta \boldsymbol{u})+\operatorname{div}(\Delta \boldsymbol{w})] \boldsymbol{I}) d v$

$-\alpha_{7} \int_{B} \operatorname{grad}(\delta \boldsymbol{w}):\left(p_{w}\left[\operatorname{grad}^{T}(\Delta \boldsymbol{u})+\frac{1-n}{n} \operatorname{div}(\Delta \boldsymbol{u}) \boldsymbol{I}\right]\right) d v$ $-\alpha_{4} \int_{B} \frac{\mu_{w}}{k} \delta \boldsymbol{w} \cdot\left[\Delta \boldsymbol{w}+\operatorname{div}(\Delta \boldsymbol{u})\left(1-\frac{1-n}{k} \frac{\partial k}{\partial n}\right) \boldsymbol{w}\right] d v$

$-\alpha_{1} \int_{B} \frac{\rho_{w}}{n} \delta \boldsymbol{w} \cdot\left[\Delta \boldsymbol{w}+\frac{2 n-1}{n} \operatorname{div}(\Delta \boldsymbol{u}) \boldsymbol{w}\right] d v$

$-\alpha_{1} \int_{B} \rho_{w} \delta \boldsymbol{w} \cdot[\Delta \boldsymbol{u}+\operatorname{div}(\Delta \boldsymbol{u}) \boldsymbol{u}] d v$

$+\alpha_{8} \int_{B} \rho_{w} \delta \boldsymbol{w} \cdot \boldsymbol{g} \operatorname{div}(\Delta \boldsymbol{u}) d v$

\section{References}

1. Arias-Trujillo, J., Blázquez, R., López-Querol, S.: A methodology based on a transfer function criterion to evaluate time integration algorithms. Soil Dynamics and Earthquake Engineering 37, 1-23 (2012)

2. Armero, F.: Formulation and finite element implementation of a multiplicative model of coupled poro-plasticity at finite strains under fully saturated conditions. Computer Methods in Applied Mechanics and Engineering 171, 205-241 (1999)

3. Arroyo, M., Ortiz, M.: Local maximum-entropy approximation schemes: a seamless bridge between finite elements and meshfree methods. International Journal for Numerical Methods in Engineering 65(13), 2167-2202 (2006) 
4. Bandara, S., Soga, K.: Coupling of soil deformation and pore fluid flow using material point method. Computers and Geotechnics 63, 199-214 (2015)

5. Biot, M.A.: Theory of propagation of elastic waves in a fluid-saturated porous solid. I. Low-Frequency range. Journal of the Acoustical Society of America 28(2), 168178 (1956)

6. Borja, R., Alarcón, E.: A mathematical framework for finite strain elastoplastic consolidation. part1: balance laws, variational formulation, and linearization. Computer Methods in Applied Mechanics and Engineering 122, 145-171 (1995)

7. Borja, R., Tamagnini, C., Alarcón, E.: Elastoplastic consolidation at finite strain. part 2: finite element implementation and numerical examples. Computer Methods in Applied Mechanics and Engineering 159, 103-122 (1998)

8. Cao, T., Sanavia, L., Schrefler, B.: A thermo-hydromechanical model for multiphase geomaterials in dynamics with application to strain localization simulation. International Journal for Numerical methods in Engineering 107, 312-337 (2016)

9. Ceccato, F., Simonini, P.: Numerical study of partially drained penetration and pore pressure dissipation in piezocone test. Acta Geotechnica 12, 195-209 (2016)

10. Cividini, A., Gioda, G.: On the dynamic analysis of two-phase soils. In: S. Pietruszczak, G.N. Pande (eds.) Proceedings of the Third International Symposium on Computational Geomechanics (ComGeo III), pp. 452$461(2013)$

11. Cuitiño, A., Ortiz, M.: A material-independent method for extending stress update algotithms from small-strain plasticity to finite plasticity with multiplicative kinematics. Engineering computations 9, 437-451 (1992)

12. Diebels, S., Ehlers, W.: Dynamic analysis of fully saturated porous medium accounting for geometrical and material non-linearities. International Journal for Numerical Methods in Engineering 39, 81-97 (1996)

13. Ehlers, W., Eipper, G.: Finite elastic deformations in liquid-saturated and empty porous solids. Transport in Porous Media 34, 179-191 (1999)

14. Gawin, D., Sanavia, L., Schrefler, B.: Cavitation modelling in saturated geomaterials with application to dynamic strain localisation. Int. J. Num. Methods in Fluids. 27, 109-125 (1998)

15. Hughes, T., Hilber, H.: Collocation, dissipation and overshoot for time integration schemes in structural dynamics. Earthquake Engineering and Structural Dynamics 6, 99-117 (1978)

16. Hughes, T.J.R., Pister, K.S.: Consistent linearization in mechanics of solids and structures. Computers and structures 8, 391-391 (1978)

17. Jeremić, B., Cheng, Z., Taiebat, M., Dafalias, Y.: Numerical simulation of fully saturated porous materials. International Journal for Numerical and Analytical Methods in Geomechanics 32, 1635-1660 (2008)

18. Lee, E.: Elastic-plastic deformation at finite strains. J. Appl. Mech. 36, 1-6 (1969)

19. Lewis, R., Schrefler, B.: The finite element method in the static and dynamic deformation and consolidation of porous media. John Wiley \& Sons Ltd. (1998)

20. Li, B., Habbal, F., Ortiz, M.: Optimal transportation meshfree approximation schemes for fluid and plastic flows. International Journal for Numerical Methods in Engineering 83, 1541-1579 (2010)

21. Li, C., Borja, R.I., Regueiro, R.A.: Dynamics or porous media at finite strain. Computed Methods in Applied Mechanics and Engineering 193, 3837-3870 (2004)
22. López-Querol, S., Blazquez, R.: Liquefaction and cyclic mobility model in saturated granular media. International Journal for Numerical and Analytical Methods in Geomechanics 30, 413-439 (2006)

23. López-Querol, S., Fernández-Merodo, J., Mira, P., Pastor, M.: Numerical modelling of dynamic consolidation on granular soils. International Journal for Numerical and Analytical Methods in Geomechanics 32, 1431-1457 (2008)

24. Marsden, J., Hughes, T.J.R.: Mathematical Foundations of Elasticity. Prentice Hall Inc., Upper Saddle River, New Jersey. (1983)

25. Mokni M., D.J.: Strain localisation measurements in undrained plane-strain biaxial tests on hostun $\mathrm{rf}$ sand. Mech. Cohes.-Frict. Mater. 4, 419-441 (1998)

26. Navas, P.: Meshfree methods applied to dynamic problems in materials in construction and soils. Ph.D. thesis, University of Castilla-La Mancha (2017)

27. Navas, P., López-Querol, S., Yu, R., Li, B.: B-bar based algorithm applied to meshfree numerical schemes to solve unconfined seepage problems through porous media. International Journal for Numerical and Analytical Methods in Geomechanics 40, 962-984 (2016)

28. Navas, P., Sanavia, L., López-Querol, S., Yu, R.: Explicit meshfree solution for large deformation dynamic problems in saturated porous media. Acta Geotechnica in print. DOI : 10.1007/s11440-017-0612-7

29. Navas, P., Yu, R., López-Querol, S., Li, B.: Dynamic consolidation problems in saturated soils solved through $\mathrm{u}-\mathrm{w}$ formulation in a LME meshfree framework. Computers and Geotechnics 79, 55-72 (2016)

30. Nemat-Nasser, S.: On finite plastic flow of crystalline solids and geomaterials. Transactions of ASME. 50, 1114-1126 (1983)

31. Ortiz, A., Puso, M., Sukumar, N.: Construction of polygonal interpolants: A maximum entropy approach. International Journal for Numerical Methods in Engineering 61(12), 2159-2181 (2004)

32. Ravichandran, N., Muraleetharan, K.: Dynamics of unsaturated soils using various finite element formulations. International Journal for Numerical and Analytical Methods in Geomechanics 33, 611-631 (2009)

33. Sanavia, L., Schrefler, B., Stein, E., Steinmann, P.: Modelling of localisation at finite inelastic strain in fluid saturated porous media. Proc. In: Ehlers W (ed.), IUTAM Symposium on Theoretical and Numerical Methods in Continuum Me- chanics of Porous Materials, Kluwer Academic Publishers pp. 239-244 (2001)

34. Sanavia, L., Schrefler, B., Steinmann, P.: A mathematical and numerical model for finite elastoplastic deformations in fluid saturated porous media. In: G. Capriz, V. Ghionna, P. Giovine (ed.) Modeling and Mechanics of Granular and Porous Materials, Series of Modeling and Simulation in Science, Engineering and Technology pp. 297-346 (2001)

35. Sanavia, L., Schrefler, B., Steinmann, P.: A formulation for an unsaturated porous medium undergoing large inelastic strains. Computational Mechanics 28, 137-151 (2002)

36. Schrefler, B., Sanavia, L., Majorana, C.: A multiphase medium model for localisation and postlocalisation simulation in geomaterials. Mech. Cohes.-Frict. Mater. 1, 95-114 (1996)

37. Simo, J.: Numerical Analysis and simulation of plasticity. In P.G. Ciarlet and J.L. Lions, eds, Numerical Methods for Solids (Part 3), vol. 6 of Handbook of Numerical Analysis. North-Holland (1998) 
38. Terzaghi, K.V.: Principles of Soil Mechanics. Engineering News-Record 95, 19-27 (1925)

39. Uzuoka, R., Borja, R.: Dynamics of unsaturated poroelastic solids at finite strain. International Journal for Numerical and Analytical Methods in Geomechanics 36, 1535-1573 (2012)

40. Wriggers, P.: Nonlinear Finite Element Methods. Springer (2008)

41. Ye, F., Goh, S., Lee, F.: A method to solve biot's $\mathrm{u}-\mathrm{u}$ formulation for soil dynamic applications using the abaqus/explicit platform. Numerical Methods in Geotechnical Engineering pp. 417-422 (2010)

42. Zhang, H., Sanavia, L., Schrefler, B.: An internal length scale in strain localisation of multiphase porous media. Mech. Cohes.-Frict. Mater. 4, 433-460 (1999)

43. Zienkiewicz, O., Chan, A., Pastor, M., Schrefler, B., Shiomi, T.: Computational Geomechanics with Special Reference to Earthquake Engineering. John Wiley, UK (1999)

44. Zienkiewicz, O., Chang, C., Bettes, P.: Drained, undrained, consolidating and dynamic behaviour assumptions in soils. Géotechnique 30(4), 385-395 (1980)

45. Zienkiewicz, O., Shiomi, T.: Dynamic behaviour of saturated porous media: The generalized biot formulation and its numerical solution. Int. J. Num. Anal. Geomech. 8, 71-96 (1984) 